\title{
Recycling of autophagosomal components from autolysosomes by recycler complex
}

\section{Yueguang Rong ( $\nabla$ rongyueguang@hust.edu.cn )}

School of Basic Medicine, Huazhong University of Science and Technology

\section{Chuchu Zhou}

School of Basic Medicine, Huazhong University of Science and Technology

\section{Zhe Wu}

School of Basic Medicine, Huazhong University of Science and Technology

\section{Wanqing Du}

School of Life Sciences, Tsinghua University

\section{Ying Li}

Tsinghua University https://orcid.org/0000-0003-4080-3214

\section{Fenglei Jian}

School of Basic Medicine, Huazhong University of Science and Technology

\section{Yufen Wang}

School of Basic Medicine, Huazhong University of Science and Technology

\section{Huilin Que}

Department of Pathogen Biology, School of Basic Medicine, Huazhong University of Science and Technology

\section{Weigang Yuan}

Department of Pathogen Biology, School of Basic Medicine, Huazhong University of Science and Technology

\section{Yuan Zhao}

Department of Pathogen Biology, School of Basic Medicine, Huazhong University of Science and Technology

\section{Rui Tian}

Department of Pathogen Biology, School of Basic Medicine, Huazhong University of Science and Technology

\section{Letter}

Keywords: ACR, STX17, SNX17, SNX5

Posted Date: November 4th, 2020 
DOI: https://doi.org/10.21203/rs.3.rs-55868/v1

License: (c) (1) This work is licensed under a Creative Commons Attribution 4.0 International License. Read Full License

Version of Record: A version of this preprint was published at Nature Cell Biology on March 24th, 2022. See the published version at https://doi.org/10.1038/s41556-022-00861-8. 


\section{Abstract}

Autolysosomes contain components both from autophagosomes and lysosomes. The contents inside the autophagosomal lumen are degraded during autophagy, while the fate of autophagosomal components on autolysosomal membrane remains unknown. Here, we found the autophagosomal membrane and transmembrane proteins are not degraded, but recycled from autolysosomes. We named this process autophagosomal components recycling (ACR). We further identified a multiprotein complex composed of SNX4, SNX5 and SNX17 essential for ACR which we termed "recycler". In this, SNX4 and SNX 5 form a heterodimer that recognizes an autophagosomal cargo STX17 and is required for generating membrane curvature on autolysosomes both via their BAR domains, to mediate the cargo sorting process. SNX17 interacts with both the dynein-dynactin complex and the SNX4-SNX5 dimer to facilitate retrograde transport of STX17. Depletion of any subunit of recycler completely blocks ACR, and also inhibits autophagy. Our discovery of ACR and identification of recycler reveal an important retrieval and recycling pathway on autolysosomes.

\section{Background}

Autophagy is a conserved process for removing damaged and unwanted materials from the cytoplasm ${ }^{1-}$ 6 . Deficiencies in autophagy are associated with many human diseases, especially neurodegenerative diseases, inflammatory disorders, and cancer ${ }^{7-9}$. During autophagy pre-autophagosomes grow and elongate by taking up membranes from multiple organelles including the $\mathrm{ER}^{10-14}$, mitochondria ${ }^{15}$, plasma membrane ${ }^{16,17}$, Golgi ${ }^{18,19}$, and ERGIC ${ }^{20-22}$. After pre-autophagosomes mature into complete autophagosomes, all the autophagosomal components are delivered to lysosomes via autophagosomelysosome fusion. The autophagosome lumenal contents are turned over in autolysosomes, while lysosomal components are recycled on the autolysosomal surface via autophagic lysosome reformation (ALR). We have previously investigated and presented details of the mechanism for the lysosomal component recycling on autolysosomes ${ }^{23-26}$. However, the fate of autophagosomal components on autolysosomes membrane remains unknown.

Here, we found that autophagosomal membrane components are recycled from autolysosomes. This process of autophagosomal components recycling (ACR) is conducted by recycler comprised of SNX4, SNX5, and SNX17. We show that SNX4 and SNX5 form heterodimers to recognize an autophagosomal cargo STX17 and generate membrane curvature on autolysosomes through their BAR domains simultaneously for cargo sorting. SNX17 functions as an adaptor, linking the SNX4-SNX5 dimer with the dynein-dynactin complex, to facilitate the cargo retrograde transport. Furthermore, we also demonstrate that recycler functions independently of the retromer and retriever complexes, and that the defect in ACR lead to inhibition of autophagy.

\section{Results}


The autophagic SNARE protein STX17, which mediates the autophagosome-lysosome fusion by forming a SNARE complex with SNAP29 and VAMP7/8, is recruited to autophagosomes and translocated to autolysosome after fusion of autophagosomes with lysosomes ${ }^{27,28}$. In agreement with these findings, we also observed that the number of autophagic vacuoles with STX17 dramatically increased after 2 hours of starvation. However, the number of STX17 puncta significantly decreased during prolonged starvation of 5 hours (Fig. 1a), suggesting the occurrence of dissociation or turn-over of STX17. Interestingly, no obvious degradation of STX17 was observed after prolonged starvation (Extended Data Fig. 1a).

In order to investigate the fate of STX17 on the surface of autolysosomes, we examined the dynamics of STX17 by time-lapse imaging in a cell line stably expressing GFP-STX17-TM and LAMP1-CR. Since GFPSTX17 blocks autophagosome-lysosome fusion and cannot be translocated to autolysosomes ${ }^{29}$, we used a GFP-tagged transmembrane domain of STX17 for this experiment. Detailed live images showed STX17 concentrated on one point of autolysosomal membrane, which then pinched off from the autolysosome (Fig. 1b). The signal intensity of STX17 on the bud was approximately 2 -fold of the signal on the autolysosome membrane (Fig. 1c), and the overall intensity of the STX17 signal on the autolysosomal membrane was reduced in a time dependent manner (Fig. 1 b and d). In contrast, the STX17 signal on existing autophagosomes remained relatively unchanged (Fig. 1d). These data suggested that STX17 on autolysosomes was not degraded to a large extent, but instead disassociated from autolysosomes through an as-of-yet uncharacterized sorting process.

Previously, we reported that lysosome components are recycled through ALR on autolysosomes ${ }^{23-26}$. To distinguish ALR from this uncharacterized sorting process, we examined both processes simultaneously in cells stably expressing CFP-LC3, GFP-STX17-TM, and LAMP1-mCherry (Fig. 1e). We found that STX17 began to be recruited to autophagosomes (labeled by LC3) after $1 \mathrm{~h}$ of starvation, and then STX17positive autophagosomes fused with lysosomes (labeled by LAMP1). Subsequently, STX17 was translocated to autolysosomes through the fusion of autophagosome with lysosomes. However, at 5 hours after starvation, the STX17 signal on the autolysosomal membrane was completely undetectable, with no ALR occurrence. At 8 hours of starvation, ALR was initiated on autolysosomes with no observable STX17 signal. This finding suggested that this uncharacterized sorting process occurs prior to ALR. Furthermore, depletion of ALR-essential genes, such as clathrin and AP2, had no effect on this sorting process (Extended Data Fig. 1b and c). These results strongly suggested that this uncharacterized sorting process is completely different from ALR and occurs before ALR on autolysosomes. We thus named this process autophagosomal components recycling (ACR).

Consistent with above results, we observed that STX17 concentrated on the acidified autolysosome membrane, indicated by lysoTracker staining, an acid dependent dye, and budded off (Fig. 1f) with STX17 showing lower signal intensity on acidified autolysosomes than non-acidified autophagosomes (Fig. 1g-i). This suggests that ACR may require acidified autophagic vacuoles. The vesicles budding off are not detectable by lysoTracker staining, despite strong positive staining occurring in the autolysosomal lumen (Fig. 1j). This suggests a lack of acidity in these vesicles. To further test if acidity is required for ACR, we treated cells with Bafilomycin A1区a lysosomal v-ATPase inhibitor, at $2 \mathrm{~h}$ after starvation to 
alkalize autolysosomes. Intriguingly, we found that all STX17 became completely invaginated into autolysosomes in vesicle form, but not on the autolysosomal membrane (Fig. 1k, l). This suggests that a high pH value not only completely blocks STX17 recycling out of autolysosomes, but further causes its invagination into the autolysosomal lumen. Together, it suggests the low $\mathrm{pH}$ value inside the autolysosome is critical for ACR.

\section{SNX4 is required for ACR}

In light of these findings, we then sought to investigate the molecular components that mechanistically participate in ACR. Based on our data above, STX17-TM contains the minimal components required for ACR, and STX17-TM with large tag at $\mathrm{N}$ terminal is delivered to autolysosomes efficiently ${ }^{29}$. Thus we employed APEX2-STX17 TM to biotinylate its functional related proteins on autolysosomes, followed by semi-quantitative mass spectrometry analysis and a screen based on protein localization and gene depletion experiments. We found that SNX4, SNX5, and SNX17 not only localize to STX17-positive autolysosomes, but their depletion leads to STX17 accumulation on autolysosomes. Subsequently, we selected SNX4, SNX5, and SNX17 for further investigation.

We first examined the role of SNX4 in ACR. In SNX4 knockdown cells, we observed that autophagosomes formed and fused with lysosomes at $2 \mathrm{~h}$ after starvation. This result suggested that SNX4 depletion exerts no negative effect on the first round of autophagosome formation and autophagosome-lysosome fusion. However, the disassociation of STX17 was completely blocked by SNX4 depletion (Fig. 2a and b), thus suggesting a critical role for SNX4 in ACR.

Previous work has shown that SNX4 recycles transferrin receptors through interaction with KIBRA on early endosomes in mammals ${ }^{30}$. We silenced KIBRA to investigate whether or not it participates in ACR, but found no effect on ACR in KIBRA-silenced cells (Extended Data Fig. 2a and Fig.2b), thus suggesting that SNX4 functions in ACR independently of the SNX4-KIBRA complex. Since SNX4 also forms heterodimers with SNX7 and SNX30 (the hypothetical homologues of snX41p and snx42p in yeast, respectively $)^{31}$, we examined if heterodimers of SNX4 with SNX7 and SNX30 are required for ACR. Depletion of SNX7 and SNX30 also caused no observable effect on ACR (Extended Data Fig. $\mathbf{2 b}$ and Fig.2b), which suggested that the SNX4-SNX7 and SNX4-SNX30 complexes are not involved in ACR. Together, these results show that SNX4 functions in ACR independent of both the SNX4-KIBRA complex as well as the SNX4-SNX7/SNX30 complex.

In order to investigate how SNX4 is involved in ACR, we further examined if SNX4 localizes to STX17positive autolysosomes. We generated a stable cell line expressing mKATE2-SNX4, GFP-STX17-TM, and LAMP1-CFP and confirmed that SNX4 did, in fact, co-localize with STX17 on the rings and buds of autolysosomes (Fig. 2C). To better understand the role of SNX4 in this sorting process, we performed time lapse imaging which revealed that STX17 concentrated on autolysosome rings and co-localized with SNX4 on the concentrated buds. At the conclusion of budding, STX17 disassociated from autolysosomes in small vesicles together with SNX4, but not with LAMP1, thus suggesting high selectivity in the sorting 
process (Fig. 2d). Since we observed SNX4 to co-localize with STX17 on the autolysosome buds, we next examined the interaction of SNX4 with STX17. We found that both endogenously- and exogenouslyexpressed SNX4 interacted with STX17 and in vitro assays demonstrated that this interaction is a direct interaction (Fig. 2e-g). Further analysis showed that SNX4 interacted with STX17 primarily through binding the transmembrane and SNARE domains, while STX17 interacted with SNX4 through binding to the BAR domain (Fig. 2h-j). Of interest, The $\mathrm{N}$ terminal domain of STX17 exerted the inhibition on its binding to SNX4 to some extent (Fig. 2h). These results suggested that SNX4 is essential for direct sorting of STX17 on autolysosomes.

\section{SNX5 cooperates with SNX4 to mediate STX17 sorting from autolysosomes}

Since SNX4-KIBRA and SNX4-SNX7/SNX30 are not involved in ACR, we sought to explore its co-players in ACR. Given BAR domain containing sorting nexins function always in pairs ${ }^{32-39}$, and the results of our initial co-localization and depletion experiment showing that SNX5, another BAR domain containing protein, also likely contributed to the sorting process, we then examined whether SNX5 was directly involved in ACR.

We found that STX17 recycling was completely and consistently blocked in SNX5 knockdown cells, confirming the role of SNX5 in ACR, but with both the first round of autophagosome formation and autophagosome-lysosome fusion remaining unaffected (Fig. 3a and b). As established in previous studies, SNX5 cooperated with the retromer complex ${ }^{33,35,38,40,41}$, but depletion of VPS35 or SNX6, two subunits of retromer complex, did not affect ACR (Extended Data Fig. 3a and Fig. 3b). This result suggested that the role of SNX5 in ACR does not rely on retromer. Similar to SNX4, SNX5 localized only to the STX17-positive buds of autolysosomes (Fig. 3c) and time lapse imaging of live cells revealed that it co-localized specifically with STX17 on these buds, disassociating from autolysosomes together with STX17 (Fig. 3d). In addition, both endogenous and exogenous SNX5 were observed to directly interact with STX17 (Fig. 3e-g). This interaction was mediated primarily by association of the SNX5 BAR domain with SNARE and transmembrane domains of the STX17 (Fig. 3h-j). These data thus indicated the direct participation of SNX5 in ACR, independent of its role in the retromer complex.

Since both SNX4 and SNX5 function in ACR and both localize to STX17-positive buds on autolysosomes, we then hypothesized that SNX4 and SNX5 were functionally connected. This possibility was supported by our finding that SNX5 exhibited significant co-localization with SNX4 on the buds of lysosomes (Fig. 3k) and that SNX5 interacted with SNX4 both in vivo and in vitro through their BAR domains (Fig.3 1-o).

Further supporting our hypothesis, SNX5 depletion blocked ACR, and concurrently led to a dramatic increase in the localization of SNX4 on STX17-positive autolysosomes in cells stably expressing mKATE2-SNX4, GFP-STX17-TM and LAMP1-CFP (Fig. 3p, q). In agreement with these results, SNX4 depletion blocked ACR and dramatically increased localization of SNX5 on STX17-positive autolysosomes in cells stably expressing mKATE2-SNX5, GFP-STX17-TM and LAMP1-CFP (Fig. 3p, q). Both results suggest: 1) SNX5 depletion blocks STX17 recycling and inhibits SNX4 disassociation, while 
SNX4 depletion blocks STX17 recycling and inhibits SNX5 disassociation; 2) the recruitment of SNX4 and SNX5 to autolysosomes is not interdependent; and 3) SNX4 overexpression cannot rescue the ACR defects resulting from SNX5 depletion, and vice versa.

In addition, the BAR domain containing proteins have been characterized for its ability to sense or induce membrane structural curvature in the generation of carrier vesicles ${ }^{42-44}$, then the ability of SNX4 and SNX5 in remodeling autolysosomal membrane was examined. Cytosols from starved wild type, SNX4 KO, SNX5 KO, and SNX17 KO cells were incubated with trypsin stripped autolysosomes purified from starved MEF cells, and then the samples were observed via scanning electron microscopy. We found that bud/tubule formation on autolysosomes was significantly inhibited for SNX4 KO and SNX5 KO cytosols, but not for wild type and SNX17 KO cytosols (Fig. 3r, s). Taken together, these data suggest SNX4 and SNX5 function in ACR interdependently, and they are not interchangeable in this process.

\section{SNX17 coordinates the interaction of STX17 with dynein-dynactin complexes to mediate ACR}

In addition to SNX4/5, the SNX-FERM family protein SNX17 was also implicated in ACR by our initial experiment showing its localization to STX17-positive autolysosomes (Fig.4a). Similar to SNX4 and SNX5, depletion of SNX17 also caused severe defects in the ACR process without affecting autophagosome formation and the first round autophagosome-lysosome fusion (Fig. 4b,c). SNX17 has also been shown to be involved in plasma membrane protein recycling together with the retriever complex ${ }^{45-47}$. However, knockdown of VPS26C and VPS35L, two retriever complex subunits, had no obvious effect on ACR (Extended Data Fig. 4a, Fig.4c), thus suggesting a retriever-independent role of SNX17 in ACR. We therefore next examined whether and how SNX17 was mechanistically involved with STX17. We subsequently found that endogenous SNX17 interacted with STX17 and that this interaction was through direct binding, as with SNX4 and SNX5 (Fig.4d-f). Mapping analysis showed that the F3 region in the FERM domain of SNX17 interacts with the SNARE and transmembrane domains of STX17, while the N terminal domain of STX17 may interfere its interaction with SNX17 (Extended Data Fig. 4b-d). Together, these cumulative data strongly suggest that SNX17 directly functions in ACR.

Since SNX17 lacks a BAR domain, and instead carries the PX and FERM domains, we speculated that SNX17 likely functions through a different mechanism than SNX4 and SNX5. To explore the mechanistic role of SNX17 in ACR, we examined its interactions with several minus end-directed transport machineries (i.e., an adaptor protein of the dynein-dynactin complex (DCTN1) and dynein heavy chain (DHC1)). Intriguingly, we found that SNX17 interacted with DCTN1, through its F3 region in the FERM domain (Fig. 4g and Extended Data Fig. 5a), and with DHC1 (Fig. 4h). Interactions were also observed between DHC1 and SNX4, SNX5, and STX17 (Fig. 4h). Furthermore, silencing DCTN1 and other subunits of the dynein dynactin complex also blocked ACR (Extended Data Fig. 5b, Fig. 4i), but not by any depletion of the endosomes or lysosome adaptor proteins HPS6, SKIP, and KIBRA (Extended Data Fig. 6a, Fig. 4i and Fig. 2b). In addition, SNX4, SNX5, and STX17 were shown to interact with DCTN1 (Extended Data Fig. 6b-d). These results suggested that SNX17 likely coordinates the retrieval of STX17 via the dynein-dynactin complex. 
Thus supporting our hypothesis, depletion of SNX17 reduced the interaction of DCTN1 with SNX4, SNX5, and STX17 (Fig.4 j-I). Consistent with these observations, suppression of SNX17 and DCTN1 significantly increased the localization of SNX4 and SNX5 on autolysosomes (Fig.4 m-o). Taken together, these results suggested that SNX17 plays a direct role in ACR by mediating the interactions between the STX17-SNX4SNX5 and the dynein-dynactin complexes.

\section{SNX4-SNX5-SNX17 forms recycler complex}

Since SNX4, SNX5, and SNX17 are all essential for successful ACR, all three co-localize on the same membrane (Fig. 5a), and all interact with STX17 (Fig. 2, Fig. 3 and Fig. 4). No additional effect on ACR defects was observed when double or triply knocking down these three genes compared to the single gene knockdown (Fig. 5b, c), indicating their shared function as a complex in ACR. To further test this hypothesis, we performed immunoprecipitation (IP) and the results were consistent with complex formation, in that each of these proteins consistently pulled down both of the other two (Fig. $\mathbf{5 d}$ ). Moreover, tandem affinity IP using SNX5, then SNX4, as successive baits clearly demonstrated that SNX4/5/17 do indeed form a stable complex (Fig. 5e).

More interestingly, overexpression of each individual gene resulted in substantially enhanced interaction between the other two proteins (e.g., SNX4 overexpression promoted SNX5-SNX17 interaction, and reciprocally true for overexpression of each gene) (Fig. 5f, g), further indicating the formation of an SNX4SNX5-SNX17 complex. In agreement with these results, in vitro binding assays also demonstrated that each of these proteins interact with the other two individually and in complex (Fig. 5h, i), suggesting the formation of a bona fide complex in vitro. Ultimately, we used a mapping strategy for in depth analysis of the binding regions that mediated the formation of this complex. This analysis showed that SNX17 interacted with the BAR domain of SNX4 and SNX5 through its F1 and F3 regions, respectively (Extended Data Fig. 7a-f). These results illustrate the mechanism by which these proteins form a complex in the ACR process.

We thus concluded that ACR required the formation of the SNX4/5/17 "recycler" complex to successfully conduct highly selective recycling of autophagosomal components. Given the severe effects on STX17 retrieval incurred by deficiency in the recycler complex, and considering the multiple roles of STX17 in autophagy $27,48-50$, we finally examined how loss of recycler function affected the overall process of autophagy. To this end, we measured the autophagic flux in SNX4, SNX5 , and SNX17 knockout cells. Our results showed that autophagic flux was strikingly inhibited in all three knock-out cells (Extended Data Fig. 7g-i). Cumulatively, these data suggest recycler defect

\section{Discussion}

Autophagosomes subsume large amounts of membrane from varied origins within a cell and fuse with lysosomes, thus leading to the highly complex autolysosomes. The lysosomal membrane components on autolysosomes are recycled through ALR, but the fate of membranes and membrane proteins from 
different cellular origins on the autophagosome surface remains elusive. Here, we describe the recycler complex, composed of SNX4, SNX5, and SNX17, which selectively sorts the autophagosomal components (e.g.STX17) out of autolysosomes, and thereby prevents its accumulation on the autolysosomal surface, independently of the retromer and retriever complexes. We thus termed this process as autophagosomal components recycling (ACR). The genetic basis of ACR differs from that of ALR and ACR occurs immediately following autophagosome-lysosome fusion, but prior to ALR. Further, loss of ACR function results in the inhibition of autophagy.

Thus far, STX17 has been the only transmembrane protein identified on the surface of completed autophagosomes. It is possible, if not likely, that other cargoes on autolysosomes are also recycled, given that multiple sources contribute to the autophagosome membrane. Ongoing research will necessarily focus on the identification of the suite of cargoes recycled from autolysosomal membranes in order to clearly determine the fate of autolysosomes. In addition, the final destination of recycling vesicles remains unclear due to technical difficulties of measurement, such as fast movement and quick running out of any focus plane, as has been observed with released STX17-carrying vesicles.

It is of particular interest that ACR occurs in a pH-dependent manner and autolysosomal alkalization results in defective ACR as well as severe invagination of STX17 into autolysosomes. We speculate that disruption of autolysosomal export may underlie this effect, resulting in the balance between export and import effectively shifting to the import, and subsequently leading to aberrant invagination of STX17. However, further experiments are necessary to clarify the mechanisms that drive this phenomenon.

SNX proteins have been reportedly involved in the sorting of diverse types and quantities of cargoes, acting in various combinations and forming different complexes. Here, we identified an uncharacterized cooperation between SNX4 and SNX5 in which they form a complex and co-localize with STX17 on the buds of autolysosomes to selectively sort STX17. In addition, both of SNX4 and SNX5 interact with STX17, through their BAR domain, suggesting that in the recycler complex SNX4 and SNX5 are responsible for cargo recognition in a similar manner to that of direct cargo recognition for ESCPE (Endosomal SNX-BAR sorting complex for promoting exit-1) related SNXs ${ }^{51-54}$. However, the the $\Phi \times \Omega \times \Phi$ recognition motif identified in several cargoes of SNX5 is not present in STX17, suggesting that an unknown motif is necessary for STX17 recognition. The region between SNX4-SNX5 at the dimer interface is unlikely to serve in this function, since this domain is necessary for interaction between the SNX proteins and inaccessible for cargo recognition. We speculate that the region between different SNX4-SNX5 dimers may instead be used for recognition since mapping analysis implied its involvement in the interaction between STX17 and SNX4 or SNX5. Further structural biology studies will provide more insight into the mechanisms underlying cargo recognition, and for STX17, in particular.

In addition, previous studies showed that SNX1/SNX2 and SNX5/SNX6 constitute the respective mammalian orthologs of yeast Vps5p and Vps17p and is therefore leads to redundancy and interchangeable between SNX1 and SNX2 ${ }^{38,39,55}$. However, here SNX4 and SNX5 are both required for ACR and not interchangeable, suggesting the discrepancy between retromer and recycler in organization. 
In this study, SNX17 was found to be essential for ACR. Although SNX17 is canonically associated with the retriever complex, retrograde trafficking of STX17 was mediated independently of SNX17 function in retriever, thus suggesting multiple roles for this protein in membrane trafficking. Minus-end motor dynein is well-known for retrograde trafficking on endosomes. We found that SNX17 localizes to autolysosomes and serves as an adaptor, connecting SNX4-SNX5 dimers with the dynein-dynactin complex, and forming a stable complex with SNX4-SNX5. These results thus strongly suggest a previously unknown complex "recycler" that has emerged for ACR on autolysosomes.

Recycling and turn-over of membranes and membrane proteins is crucial for maintaining organelle identity and normal function. In this study, we identified a multi-protein recycler complex (SNX4-SNX5SNX17) that performs a recycling process for autophagosomal components on autolysosomes, independent of other retrieval processes. Further study is warranted to identify other machineries and regulators as part of the comprehensive understanding of the fate determination of autolysosomes, as well as maintenance of the identity and function of autophagosomes and lysosomes. In addition, given that SNX4 and SNX5 have been implicated in many human diseases, closer scrutiny of ACR and recycler may reveal if their dysfunction is involved in SNX-related diseases.

\section{Methods}

\section{Reagents and antibodies}

Reagents used in this study were as follows: Earle's balanced salt solution (Sigma, E2888), LysoTracker ${ }^{\text {TM }}$ Red (Invitrogen, L7528), Bafilomycin A1 (Sigma, 19148), MG132 (MCE, HY-13259), chloroquine (Sigma, C6628), ANTI-FLAG M2 Affinity Gel (Sigma, A2220), Anti-HA Affinity Gel (Sigma, A2095), Glutathione Sepharose ${ }^{\circledR}$ 4B glutathione-sepharose Resin (GE, 17-0756-04), Ni-NTA agarose resin (GE healthcare, 175318-06). 


\begin{tabular}{|c|c|c|}
\hline REAGENT or RESOURCE & SOURCE & IDENTIFIER \\
\hline \multicolumn{3}{|l|}{ Antibodies } \\
\hline Rabbit polyclonal anti-SNX4 & Proteintech & Cat\#11506-1-AP \\
\hline Rabbit monoclonal anti-SNX5 & Abcam & Cat\#ab180520 \\
\hline Rabbit polyclonal anti-SNX17 & Proteintech & Cat\#10275-1-AP \\
\hline Mouse monoclonal anti-a-Tubulin & Proteintech & Cat\#66031-1-lg \\
\hline Rabbit polyclonal anti-Flag & Sangon Biotech & Cat\#D110005 \\
\hline Mouse monoclonal anti-Flag M2 & Sigma & Cat\#F3165 \\
\hline Rabbit polyclonal anti-HA & Sangon Biotech & Cat\#D110004 \\
\hline Rabbit polyclonal anti-HA & Sigma-Aldrich & Cat\#H6908 \\
\hline Rabbit monoclonal anti-Myc & $\begin{array}{l}\text { Santa Cruz } \\
\text { Biotechnology }\end{array}$ & Cat\#SC-40 \\
\hline Rabbit polyclonal anti-LAMP1 & Sigma & Cat\#L1418 \\
\hline Rabbit polyclonal anti-LC3 & Sigma & Cat\#L7543 \\
\hline Rabbit polyclonal anti-DHC1 & Proteintech & Cat\#12345-1-AP \\
\hline Mouse monoclonal anti-DYNLL1 & $\begin{array}{l}\text { Santa Cruz } \\
\text { Biotechnology }\end{array}$ & Cat\# sc-136287 \\
\hline Rabbit monoclonal anti-DCTN1 & $\begin{array}{l}\text { Cell Signaling } \\
\text { Technology }\end{array}$ & Cat\#69399T \\
\hline Rabbit polyclonal anti-Actin & Service Bio & Cat\# GB11001 \\
\hline Rabbit polyclonal anti- $6 \times \mathrm{His}$ & Sangon Biotech & D110002 \\
\hline Mouse monoclonal anti-SQSTM1/p62 & $\begin{array}{l}\text { Cell Signaling } \\
\text { Technology }\end{array}$ & Cat\# 88588S \\
\hline $\begin{array}{l}\text { Fluorescein (FITC) AffiniPure Goat Anti-Mouse IgG } \\
(\mathrm{H}+\mathrm{L})\end{array}$ & Jackson & $\begin{array}{l}\text { Cat\#115-095- } \\
003\end{array}$ \\
\hline $\begin{array}{l}\text { Fluorescein (FITC) AffiniPure Goat Anti-Rabbit IgG } \\
(\mathrm{H}+\mathrm{L})\end{array}$ & Jackson & $\begin{array}{l}\text { Cat\#111-095- } \\
003\end{array}$ \\
\hline $\mathrm{Cy}^{\text {TM}} 3$ AffiniPure Goat Anti-Mouse lgG $(\mathrm{H}+\mathrm{L})$ & Jackson & $\begin{array}{l}\text { Cat\#115-165- } \\
003\end{array}$ \\
\hline $\mathrm{Cy}^{\text {TM}} 3$ AffiniPure Goat Anti-Rabbit lgG $(\mathrm{H}+\mathrm{L})$ & Jackson & $\begin{array}{l}\text { Cat\#111-165- } \\
003\end{array}$ \\
\hline Goat Anti-Mouse lgG(H+L)-HRP & SouthernBiotech & Cat\#1036-05 \\
\hline Goat Anti-Rabbit IgG-HRP & SouthernBiotech & $\begin{array}{l}\text { Cat\# SBA4030- } \\
05\end{array}$ \\
\hline
\end{tabular}




\section{Cell culture and transfection}

HEK293T, U2OS and Hela cells were cultured in DMEM (Hyclone) supplemented with 10\% FBS (Gibco) and $1 \%$ Penicillin-Streptomycin Solution (Beyotime) at $37^{\circ} \mathrm{C}$ with $5 \% \mathrm{CO} 2$. For starvation treatment, cells were washed three times with PBS (Hyclone) and then incubated with EBSS for the indicated time. Hela cells were transiently transfected using Lipofectamine 3000 (Invitrogen) according to the manufacturer's instructions. Transient transfection of plasmid in HEK293T cells was performed using PEI according to the manufacturer's protocol . Cells were analyzed $24 \mathrm{~h}$ after transfection. For RNA interference, siRNA duplexes were transfected into cells using Lipofectamine RNAiMAX (Invitrogen) according to the manufacturer's instructions, $48 \mathrm{~h}$ after transfection, cells were harvested for analysis.

\section{siRNA sequences}

For siRNA-mediated gene knockdown, siRNA duplexes were purchased from GenePharma. Cells were analyzed $48 \mathrm{~h}$ after transfection of the siRNA. The targeting sequences were: 


\begin{tabular}{|c|c|}
\hline SiRNA & siRNA Sequence \\
\hline NC & UUCUUCGAACGUGUCACGUTT \\
\hline SNX4-1 & CGAACTGGAAGAAATGCCATGAACA \\
\hline SNX4-2 & CAGCTGAAGGCAGACTCCAGGTTAA \\
\hline SNX5 & CUACGAAGCCCGACUUUGA \\
\hline SNX6 & UAAAUCAGCAGAUGGAGUA \\
\hline SNX7-1 & GCAGGAAGGCTTTACATAA \\
\hline SNX7-2 & GAATTTGACTCCAGTGAAT \\
\hline SNX17 & CCAGUGAUGUCCACGGCAA \\
\hline SNX30-1 & GGAUCAUCAAAGAAGAAAU \\
\hline SNX30-2 & CCGAGAAGUUUGUGGUAAA \\
\hline SNX30-3 & CCUGUGCUCAGGGAAUAUA \\
\hline KIBRA-1 & AGCACGAGCTGCAGTTCAA \\
\hline KIBRA-2 & CCGCTCACCTTTGCTGACT \\
\hline DYNLL1a & AUGCGGACAUGUCGGAAGA \\
\hline DYNLL1b & AACAAGGACUGCAGCCUAA \\
\hline DHC1a & ACAUCAACAUAGACAUUCA \\
\hline DHC1b & CCAAGCAGAUAAGGCAAUA \\
\hline DCTN1a & GAGAGGGAACUGAUACAACTT \\
\hline DCTN1b & GGAACGCUAUAUGGAGGAGTT \\
\hline HPS6-1 & CAGGGUACAUCUGCUAGAA \\
\hline HPS6-2 & CCCAGUUCUAAGCCCAUAU \\
\hline HPS6-3 & GGUCUCUCCUACAGUAAGA \\
\hline VPS35 & AUUUGGUGCGCCUCAGUCATT \\
\hline SKIP & CUUCUGAACUGGACCGAUUTT \\
\hline VPS35L-1 & ACAAUGCCUUGCUGUUGAA \\
\hline VPS35L-2 & AUCGUUCCUUCUGGAAUUC \\
\hline VPS26C-1 & GUAACAAAGUUCUGUAUGA \\
\hline VPS26C-2 & CAAACUGUGUCAUCACGCA \\
\hline
\end{tabular}

Page 13/39 


\begin{tabular}{|ll|} 
Clathrin & AACCUGCGGUCUGGAGUCAAC \\
AP2a & AAGAGCAUGUGCACGCUGGCCATT \\
\hline
\end{tabular}

\section{Immunostaining assays}

Cells grown on coverslips were washed with PBS and fixed in 4\% paraformaldehyde in PBS for $15 \mathrm{~min}$ at room temperature. After washing three times with PBS, Cells were permeabilized with $0.1 \%$ saponin in PBS for 10 min, blocked with $10 \%$ goat serum in PBS for $1 \mathrm{~h}$.Then , the cells were incubated with primary antibodies for $1 \mathrm{~h}$ or overnight at $4{ }^{\circ} \mathrm{C}$. After washing three times with PBS, cells were incubated with appropriate secondary antibodies at room temperature for $1 \mathrm{~h}$, and washed three times with PBS. The slides were mounted and images were acquired under a laser scanning confocal microscope (FV3000, Olympus).

\section{Immunoprecipitation assays}

HEK293T cells were washed in ice-cold PBS and lysed in lysis buffer (20 mM Tris-HCl, pH 7.5, $150 \mathrm{mM}$ $\mathrm{NaCl}, 1 \mathrm{mM}$ EDTA, $0.5 \% \mathrm{NP}-40,1 \mathrm{ug} / \mathrm{ml}$ Aprotinin, $1 \mathrm{ug} / \mathrm{ml}$ Pepstatin A, 1ug/ml Leupeptin, 200mM NaF, $200 \mathrm{mM} \mathrm{Na}_{3} \mathrm{VO}_{3}$ ) for $10 \mathrm{~min}$ at $4^{\circ} \mathrm{C}$. After centrifugation at $12,000 \mathrm{~g}$ for $10 \mathrm{~min}$ at $4{ }^{\circ} \mathrm{C}$, the supernatants were subjected to immunoprecipitation using anti-Flag M2 or anti-HA Affinity Gel for $12 \mathrm{~h}$ at $4^{\circ} \mathrm{C}$. Precipitated immunocomplexes were washed three times in lysis buffer and boiled in $2 x$ sample buffer. Then samples were subjected to SDS-PAGE and analyzed by immunoblot..

\section{Western Blotting}

Cells were lysed by sodium dodecyl sulphate (SDS) buffer. Samples extracted from cells were subjected to SDS-PAGE electrophoresis and immobilized on a polyvinylidene fluoride (PVDF) membrane (BIO-RAD, 162-0177). After blocking non-specific proteins on PVDF membrane by $5 \%$ skim milk for $1 \mathrm{~h}$ at room temperature, target proteins were probed with specific primary antibodies for $12 \mathrm{~h}$ at $4^{\circ} \mathrm{C}$ and then incubated with secondary antibody conjugated with Horseradish Peroxidase for $1 \mathrm{~h}$ at room temperature. Finally, visualization was carried out using enhanced chemiluminescence(Beyotime, P0018M-2) according to the manufacturer's protocol.

\section{In vitro binding assay}

Genes were cloned into pGEX-4T-1 or pET-28a vector for expression in E. coli BL21 (DE3). The recombinant proteins were purified by glutathione-sepharose resin or Ni-affinity resin. In GST pull-down assays, GST and GST tagged proteins were applied to GST resin, then were incubated with $1 \mu \mathrm{g}$ Histagged proteins in binding buffer (20mM Tris-HCl, $\mathrm{pH} 7.4,150 \mathrm{mM} \mathrm{NaCl}, 1 \mathrm{mM}$ EDTA, 0.5\% NP40) supplemented with protease inhibitor cocktail (Roche) for $2 \mathrm{~h}$ at $4^{\circ} \mathrm{C}$. After three washes, proteins were eluted and dissolved in sample buffer for SDS-PAGE and immunoblotting.

\section{Live cell imaging}


Cells were placed on a four-chambered cover glass (In vitro scientific, syD35-20-1-N) 1 day before observation. During live imaging, the culture dish was mounted on an inverted microscope (Olympus, FV3000) to maintain incubation conditions at $37^{\circ} \mathrm{C}$ and $5 \% \mathrm{CO} 2$ using a Plan Apochromat N 100x/1.70 oil. Images or videos were recorded using confocal laser microscope system, then further processed and analysed using ImageJ.

\section{Cytosol preparation}

The cells were cultured to confluence and starved in EBSS for 2 hours. Then the cells were harvested by scraping and centrifuging at $600 \times \mathrm{g}$ for $5 \mathrm{~min}$, washed with PBS followed by another $600 \times \mathrm{g}$-spin for $5 \mathrm{~min}$ and homogenized by passing through a $22 \mathrm{G}$ needle in a $1 \times$ cell pellet volume of extraction buffer ( 50 mM HEPES-KOH, pH 7.4, 500mM sucrose, 2mM PMSF, 4 mM EDTA and 4 mM EGTA) plus cocktail protease inhibitors (Roche, Indianapolis, IN), phosphatase inhibitors (Roche) and $0.3 \mathrm{mM}$ DTT. The cell homogenates were centrifuged at $160,000 \times \mathrm{g}$ for $30 \mathrm{~min}$, supernatant fractions were collected and the centrifugation was repeated three times to achieve a clarified fraction.

\section{FEISEM}

For FEISEM, lysosome isolation was performed with a lysosome isolation kit (Sigma-Aldrich) according to the manufacturer's manual. And cytosols were incubated with trypsin stripped autolysosomes on glass chips at $37^{\circ}$ for $1 \mathrm{~h}$, and then fixed with $2 \%$ glutaraldehyde in PBS buffer at room temperature for 30 min. Then, the samples were rinsed and post-fixed with $1.0 \% \mathrm{OsO}_{4}$ in $0.1 \mathrm{M}$ sodium cacodylate buffer at room temperature for $20 \mathrm{~min}$. After dehydration in a graded series of ethanol $(30 \%, 50 \%, 70 \%, 90 \%, 100 \%, 100 \%$, 10 min each), the samples were transferred to Arklone for critical-point drying using highest-purity $\mathrm{CO}_{2}$ in

a Hitachi HCP-2 Critical Point Dryer. The samples were then coated with $4 \mathrm{~nm}$ gold in a Hitachi E-1045 ion sputter coater and viewed in a Hitachi scanning electron microscope $\$ 4800$ at a $6 \mathrm{kV}$ accelerating voltage.

\section{Statistical analyses}

Statistical analysis was carried out on the data from independent experiments using Prism software (GraphPad). The error bars in the figures represent SEM; the $n$ value is specified in the legends.

\section{Data availability}

The authors declare that all relevant data supporting the findings of this study are available within the paper and its supplementary information files. Additional information including raw data are available from the corresponding author upon reasonable request.

\section{Declarations}

\section{Acknowledgements}


The work was supported by grants from NSFC (91854116 and 31771529) and the Junior Thousand Talents Program of China (to Y.R.). The work was partially supported by HUST COVID-19 Rapid Response Call.

\section{Author Contributions}

Y.R., C.Z. and Z.W. conceived and designed the experiments. C.Z. and Z.W. performed the biological and biochemical experiments. W.D. carried out the in vitro experiments. C.Z., Z.W., W.D. and Y.R. analyzed the data and wrote the manuscript with the help of all authors.

\section{Competing interests}

The authors declare no competing interests.

\section{References}

1 Mizushima, N. The ATG conjugation systems in autophagy. Curr Opin Cell Bio/63, 1-10, doi:10.1016/j.ceb.2019.12.001 (2019).

2 Mizushima, N., Yoshimori, T. \& Ohsumi, Y. The role of Atg proteins in autophagosome formation. Annu Rev Cell Dev Bio/27, 107-132, doi:10.1146/annurev-cellbio-092910-154005 (2011).

$3 \mathrm{Yu}$, L., Chen, Y. \& Tooze, S. A. Autophagy pathway: Cellular and molecular mechanisms. Autophagy14, 207-215, doi:10.1080/15548627.2017.1378838 (2018).

4 Dikic, I. \& Elazar, Z. Mechanism and medical implications of mammalian autophagy. Nat Rev Mol Cell Bio/19, 349-364, doi:10.1038/s41580-018-0003-4 (2018).

5 Bento, C. F. et al. Mammalian Autophagy: How Does It Work? Annu Rev Biochem85, 685-713, doi:10.1146/annurev-biochem-060815-014556 (2016).

6 Zhao, Y. G. \& Zhang, H. Core autophagy genes and human diseases. Curr Opin Cell Bio/61, 117-125, doi:10.1016/j.ceb.2019.08.003 (2019).

7 Yang, Y. \& Klionsky, D. J. Autophagy and disease: unanswered questions. Cell Death Differ27, 858-871, doi:10.1038/s41418-019-0480-9 (2020).

8 Levine, B. \& Kroemer, G. Biological Functions of Autophagy Genes: A Disease Perspective. Cel/176, 1142, doi:10.1016/j.cell.2018.09.048 (2019).

9 Poillet-Perez, L. \& White, E. Role of tumor and host autophagy in cancer metabolism. Genes De133, 610619, doi:10.1101/gad.325514.119 (2019).

10 Axe, E. L. et al. Autophagosome formation from membrane compartments enriched in phosphatidylinositol 3-phosphate and dynamically connected to the endoplasmic reticulum. J Cell 
Bio/182, 685-701, doi:10.1083/jcb.200803137 (2008).

11 Hayashi-Nishino, M. et al. A subdomain of the endoplasmic reticulum forms a cradle for autophagosome formation. Nat Cell Bio/11, 1433-1437, doi:10.1038/ncb1991 (2009).

12 Yla-Anttila, P., Vihinen, H., Jokitalo, E. \& Eskelinen, E. L. 3D tomography reveals connections between the phagophore and endoplasmic reticulum. Autophagy5, 1180-1185, doi:10.4161/auto.5.8.10274 (2009).

13 Graef, M., Friedman, J. R., Graham, C., Babu, M. \& Nunnari, J. ER exit sites are physical and functional core autophagosome biogenesis components. Mol Biol Cel/24, 2918-2931, doi:10.1091/mbc.E13-07-0381 (2013).

14 Zoppino, F. C., Militello, R. D., Slavin, I., Alvarez, C. \& Colombo, M. I. Autophagosome formation depends on the small GTPase Rab1 and functional ER exit sites. Traffic11, 1246-1261, doi:10.1111/j.1600-0854.2010.01086.x (2010).

15 Hailey, D. W. et al. Mitochondria supply membranes for autophagosome biogenesis during starvation. Cel/141, 656-667, doi:10.1016/j.cell.2010.04.009 (2010).

16 Ravikumar, B., Moreau, K., Jahreiss, L., Puri, C. \& Rubinsztein, D. C. Plasma membrane contributes to the formation of pre-autophagosomal structures. Nat Cell Bio/12, 747-757, doi:10.1038/ncb2078 (2010).

17 Moreau, K., Ravikumar, B., Renna, M., Puri, C. \& Rubinsztein, D. C. Autophagosome precursor maturation requires homotypic fusion. Cel/146, 303-317, doi:10.1016/j.cell.2011.06.023 (2011).

18 Geng, J., Nair, U., Yasumura-Yorimitsu, K. \& Klionsky, D. J. Post-Golgi Sec proteins are required for autophagy in Saccharomyces cerevisiae. Mol Biol Cel/21, 2257-2269, doi:10.1091/mbc.E09-11-0969 (2010).

19 Guo, Y. et al. AP1 is essential for generation of autophagosomes from the trans-Golgi network. J Cell Sci125, 1706-1715, doi:10.1242/jcs.093203 (2012).

20 Ge, L., Melville, D., Zhang, M. \& Schekman, R. The ER-Golgi intermediate compartment is a key membrane source for the LC3 lipidation step of autophagosome biogenesis. Elife2, e00947, doi:10.7554/eLife.00947 (2013).

21 Ge, L., Zhang, M. \& Schekman, R. Phosphatidylinositol 3-kinase and COPIl generate LC3 lipidation vesicles from the ER-Golgi intermediate compartment. Elife3, e04135, doi:10.7554/eLife.04135 (2014).

$22 \mathrm{Ge}$, L. et al. Remodeling of ER-exit sites initiates a membrane supply pathway for autophagosome biogenesis. EMBO Rep18, 1586-1603, doi:10.15252/embr.201744559 (2017). 
$23 \mathrm{Yu}$, L. et al. Termination of autophagy and reformation of lysosomes regulated by mTOR. Nature465, 942-946, doi:10.1038/nature09076 (2010).

24 Rong, Y. et al. Clathrin and phosphatidylinositol-4,5-bisphosphate regulate autophagic lysosome reformation. Nat Cell Bio/14, 924-934, doi:10.1038/ncb2557 (2012).

25 Rong, Y. et al. Spinster is required for autophagic lysosome reformation and mTOR reactivation following starvation. Proc Natl Acad Sci U S A108, 7826-7831, doi:10.1073/pnas.1013800108 (2011).

26 Du, W. et al. Kinesin 1 Drives Autolysosome Tubulation. Dev Cel/37, 326-336, doi:10.1016/j.devcel.2016.04.014 (2016).

27 Itakura, E., Kishi-Itakura, C. \& Mizushima, N. The hairpin-type tail-anchored SNARE syntaxin 17 targets to autophagosomes for fusion with endosomes/lysosomes. Cel/151, 1256-1269, doi:10.1016/j.cell.2012.11.001 (2012).

28 Takats, S. et al. Autophagosomal Syntaxin17-dependent lysosomal degradation maintains neuronal function in Drosophila. J Cell Bio/201, 531-539, doi:10.1083/jcb.201211160 (2013).

29 Uematsu, M., Nishimura, T., Sakamaki, Y., Yamamoto, H. \& Mizushima, N. Accumulation of undegraded autophagosomes by expression of dominant-negative STX17 (syntaxin 17) mutants. Autophagy13, 14521464, doi:10.1080/15548627.2017.1327940 (2017).

30 Traer, C. J. et al. SNX4 coordinates endosomal sorting of TfnR with dynein-mediated transport into the endocytic recycling compartment. Nat Cell Bio/9, 1370-1380, doi:10.1038/ncb1656 (2007).

31 van Weering, J. R. et al. Molecular basis for SNX-BAR-mediated assembly of distinct endosomal sorting tubules. EMBO J31, 4466-4480, doi:10.1038/emboj.2012.283 (2012).

32 Bonifacino, J. S. \& Hurley, J. H. Retromer. Curr Opin Cell Bio/20, 427-436, doi:10.1016/j.ceb.2008.03.009 (2008).

33 Cullen, P. J. Endosomal sorting and signalling: an emerging role for sorting nexins. Nat Rev Mol Cell Bio/9, 574-582, doi:10.1038/nrm2427 (2008).

34 Wang, J. et al. Endosomal receptor trafficking: Retromer and beyond. Traffic19, 578-590, doi:10.1111/tra.12574 (2018).

35 Cullen, P. J. \& Steinberg, F. To degrade or not to degrade: mechanisms and significance of endocytic recycling. Nat Rev Mol Cell Bio/19, 679-696, doi:10.1038/s41580-018-0053-7 (2018).

$36 \mathrm{Ma}, \mathrm{M}$. \& Burd, C. G. Retrograde trafficking and plasma membrane recycling pathways of the budding yeast Saccharomyces cerevisiae. Traffic21, 45-59, doi:10.1111/tra.12693 (2020). 
37 Weeratunga, S., Paul, B. \& Collins, B. M. Recognising the signals for endosomal trafficking. Curr Opin Cell Bio/65, 17-27, doi:10.1016/j.ceb.2020.02.005 (2020).

38 Wassmer, T. et al. The retromer coat complex coordinates endosomal sorting and dynein-mediated transport, with carrier recognition by the trans-Golgi network. Dev Cel/17, 110-122, doi:10.1016/j.devcel.2009.04.016 (2009).

39 Rojas, R., Kametaka, S., Haft, C. R. \& Bonifacino, J. S. Interchangeable but essential functions of SNX1 and SNX2 in the association of retromer with endosomes and the trafficking of mannose 6-phosphate receptors. Mol Cell Bio/27, 1112-1124, doi:10.1128/MCB.00156-06 (2007).

40 Wassmer, T. et al. A loss-of-function screen reveals SNX5 and SNX6 as potential components of the mammalian retromer. J Cell Sci120, 45-54, doi:10.1242/jcs.03302 (2007).

41 Chen, K. E., Healy, M. D. \& Collins, B. M. Towards a molecular understanding of endosomal trafficking by Retromer and Retriever. Traffic20, 465-478, doi:10.1111/tra.12649 (2019).

42 Peter, B. J. et al. BAR domains as sensors of membrane curvature: the amphiphysin BAR structure. Science303, 495-499, doi:10.1126/science.1092586 (2004).

43 McMahon, H. T. \& Gallop, J. L. Membrane curvature and mechanisms of dynamic cell membrane remodelling. Nature438, 590-596, doi:10.1038/nature04396 (2005).

44 Suetsugu, S., Toyooka, K. \& Senju, Y. Subcellular membrane curvature mediated by the BAR domain superfamily proteins. Semin Cell Dev Bio/21, 340-349, doi:10.1016/j.semcdb.2009.12.002 (2010).

45 van Kerkhof, P. et al. Sorting nexin 17 facilitates LRP recycling in the early endosome. EMBO J24, 2851-2861, doi:10.1038/sj.emboj.7600756 (2005).

46 Steinberg, F., Heesom, K. J., Bass, M. D. \& Cullen, P. J. SNX17 protects integrins from degradation by sorting between lysosomal and recycling pathways. J Cell Bio/197, 219-230, doi:10.1083/jcb.201111121 (2012).

$47 \mathrm{McNally}, \mathrm{K}$. E. et al. Retriever is a multiprotein complex for retromer-independent endosomal cargo recycling. Nat Cell Bio/19, 1214-1225, doi:10.1038/ncb3610 (2017).

48 Hamasaki, M. et al. Autophagosomes form at ER-mitochondria contact sites. Nature495, 389-393, doi:10.1038/nature11910 (2013).

49 Kumar, S. et al. Mechanism of Stx17 recruitment to autophagosomes via IRGM and mammalian Atg8 proteins. J Cell Bio/217, 997-1013, doi:10.1083/jcb.201708039 (2018).

50 Kumar, S. et al. Phosphorylation of Syntaxin 17 by TBK1 Controls Autophagy Initiation. Dev Cel/49, 130-144 e136, doi:10.1016/j.devcel.2019.01.027 (2019). 
51 Kvainickas, A. et al. Cargo-selective SNX-BAR proteins mediate retromer trimer independent retrograde transport. J Cell Bio/216, 3677-3693, doi:10.1083/jcb.201702137 (2017).

52 Simonetti, B., Danson, C. M., Heesom, K. J. \& Cullen, P. J. Sequence-dependent cargo recognition by SNX-BARs mediates retromer-independent transport of CI-MPR. J Cell Bio/216, 3695-3712, doi:10.1083/jcb.201703015 (2017).

53 Elwell, C. A. et al. Chlamydia interfere with an interaction between the mannose-6-phosphate receptor and sorting nexins to counteract host restriction. Elife6, doi:10.7554/eLife.22709 (2017).

54 Simonetti, B. et al. Molecular identification of a BAR domain-containing coat complex for endosomal recycling of transmembrane proteins. Nat Cell Bio/21, 1219-1233, doi:10.1038/s41556-019-0393-3 (2019).

55 Griffin, C. T., Trejo, J. \& Magnuson, T. Genetic evidence for a mammalian retromer complex containing sorting nexins 1 and 2. Proc Natl Acad Sci U S A102, 15173-15177, doi:10.1073/pnas.0409558102 (2005).

\section{Figures}




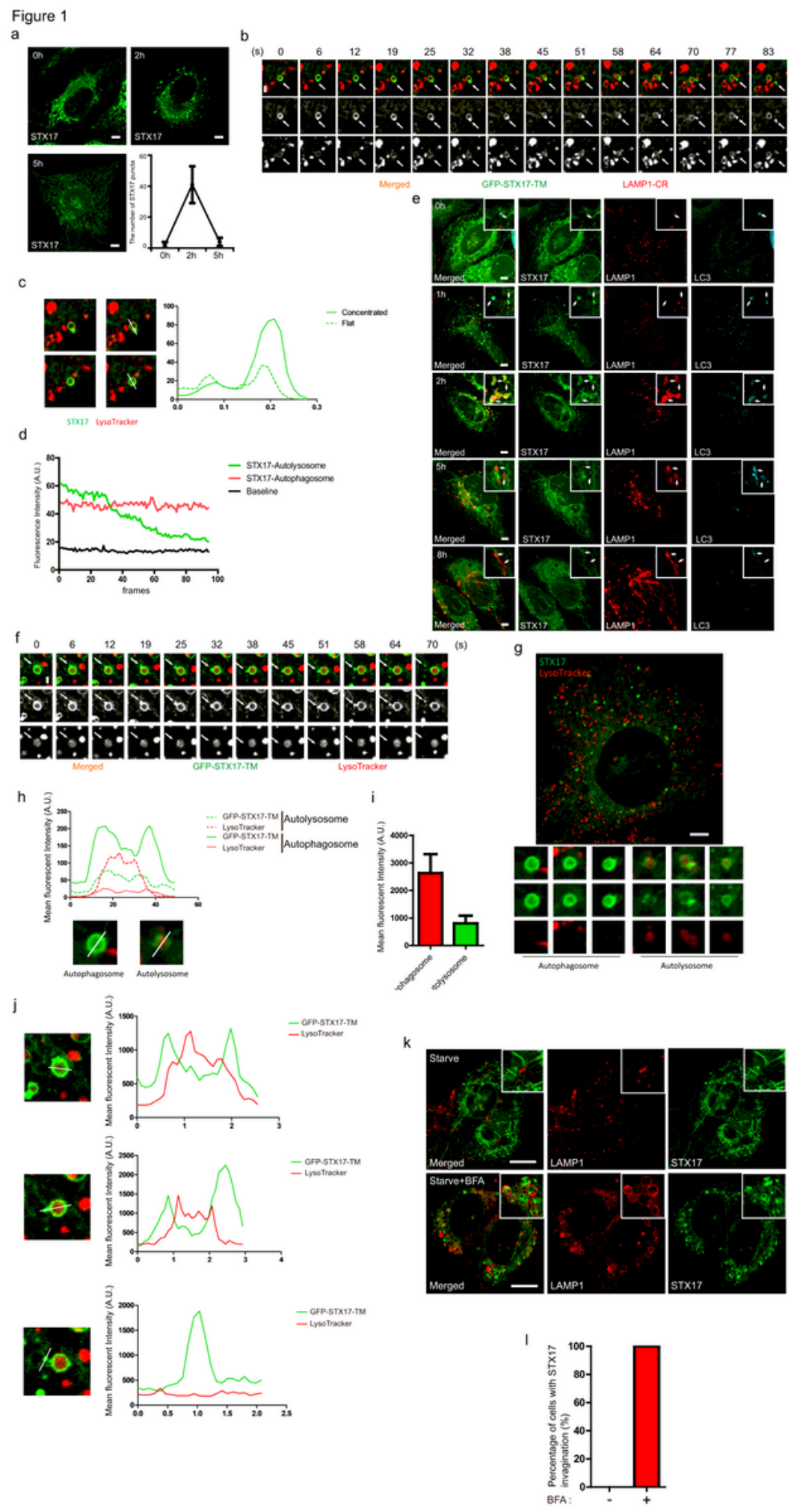

Figure 1

STX17 is sorted from autolysosomes by autophagic component recycling (ACR) a, The dynamic changes in STX17 puncta number during autophagy. U2OS cells stably expressing Flag-STX17 were starved with EBSS for the indicated hours and stained with antibodies against Flag. Scale bar, $5 \mu \mathrm{m}$. Quantification of STX17 puncta (right bottom). Data are means \pm SEM for 100 cells. b, STX17 was sorted from autolysosomes. U2OS cells stably expressing GFP-STX17-TM, LAMP1-mCherry was starved for $2 \mathrm{~h}$ with 
EBSS and time-lapse images were taken $6.4 \mathrm{~s} /$ frame. Selected frames were shown as indicated time points. Scale bar, $1 \mu \mathrm{m}$. c, STX17 was concentrated on autolysosomal buds. The fluorescent signal of STX17 on autolysosomes from selected images in c was plotted. $d$, The signal intensity of STX17 on autolysosomes decreased during autophagy. The fluorescent intensity of STX17 on the autophagosome and the autolysosome was quantified. e, STX17 disassociation from autolysosomes occurs before ALR. U2OS cells stably expressing GFP-STX17-TM, LAMP1-mCherry and CFP-LC3 were starved with EBSS for the indicated hours. Scale bar, $5 \mu \mathrm{m}$. $\mathrm{f}$, The dynamics of STX17 sorting on acidified autolysosomes. U2OS cells stably expressing GFP-STX17-TM were starved with EBSS for $2 \mathrm{~h}$ and stained with lysoTracker. Timelapse images were taken at $6.4 \mathrm{~s} /$ frame. Selected frames were shown as indicated time points. Scale bar, $1 \mu \mathrm{m} . \mathrm{g}$, The intensity of STX17 on autolysosomes is lower than on autophagosomes. U2OS cells stably expressing GFP-STX17-TM were starved with EBSS and stained with lysoTracker for $2 \mathrm{~h}$. Scale bar, $1 \mu \mathrm{m}$. h, Negative correlation between fluorescent intensity of STX17 and pH of autolysosomes. Fluorescent intensity of STX17 and lysoTracker on autophagosomes and autolysosomes in g were analyzed. i, Fluorescent intensity of STX17 on autophagosomes and autolysosomes was quantified. $n=50$. j, The relative fluorescent intensity of STX17 and lysoTracker on autolysosomes and newly generated STX17 vesicles. Selected images from $f$ were analyzed. $k$, The sorting of STX17 out of autolysosomes was blocked by Bafilomycin A1. U20S cells stably expressing GFP-STX17-TM and LAMP1-mCherry were starved with EBSS for $2 \mathrm{~h}$. After $2 \mathrm{~h}$ of starvation, cells were treated with or without Bafilomycin $\mathrm{A} 1 \mathrm{for}$ another $6 \mathrm{~h}$. Scale bar, $5 \mu \mathrm{m}$. I, Percentage of cells with STX17 invagination into autolysosomes. Images in $\mathrm{k}$ were analyzed. Data are means \pm SEM for 100 cells. 


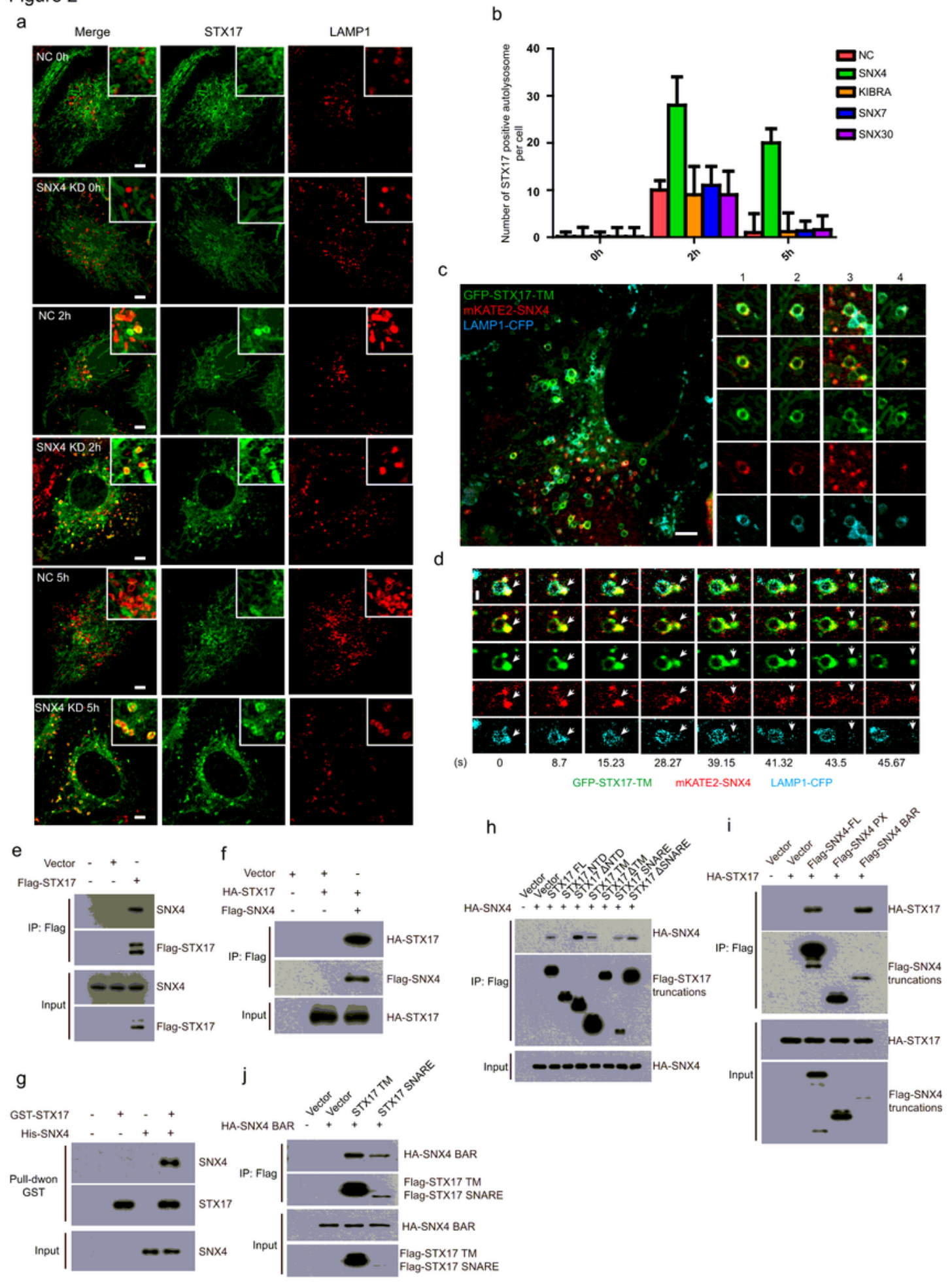

\section{Figure 2}

SNX4 is required for ACR a, SNX4 depletion leads to defects in ACR. U2OS cells stably expressing FlagSTX17 were transfected with non-targeting siRNA (NC) or siRNA against SNX4. Forty-eight hours after transfection cells are starved with EBSS for the indicated hours. Scale bar, $5 \mu \mathrm{m}$. b, Quantification of STX17-positive autolysosome number. Images in a and extended data figure $2 \mathrm{a}$ and b were analyzed. Data are means \pm SEM for 100 cells. c, SNX4 localizes to autolysosomes. U2OS cells stably expressing 
GFP-STX17-TM, mKATE2-SNX4 and LAMP1-CFP were starved with EBSS for $2 \mathrm{~h}$ and live images were taken. Scale bar, $5 \mu \mathrm{m}$. d, Selected frames from time-lapse movies of starved U2OS cells. U2OS cells stably expressing GFP-STX17-TM, mKATE2-SNX4, and LAMP1-CFP were starved with EBSS for 2h and movies were taken. Scale bar, $1 \mu \mathrm{m}$. e, Endogenous SNX4 interacted with STX17. HEK293T cells were transfected with empty vector or Flag-STX17. Twenty-four hours after transfection, cells were lysed and immunoprecipitated with anti-Flag antibody. Immunoblotting was performed with indicated antibodies. $f$, SNX4 interacted with STX17. HEK293T cells were transfected with empty vector, HA-STX17 with or without Flag-SNX4. Twenty-four hours after transfection, cells were lysed and immunoprecipitated with anti-Flag antibody. Immunoblotting was performed with indicated antibodies. g, Direct binding of SNX4 with STX17 in vitro. Glutathione Sepharose beads bound with GST or GST-STX17 were incubated with purified His-SNX4 for 16h, and then eluted for immunoblotting. h, SNX4 interacted with the transmembrane and SNARE domains of STX17. HEK293T cells were transfected with empty vector, HASNX4 with or without truncated variants of STX17. Twenty-four hours after transfection, cells were lysed and immunoprecipitated with anti-Flag antibody. Immunoblotting was performed with the indicated antibodies. i, STX17 interacts with the BAR domain of SNX4. HEK293T cells were transfected with empty vector, HA-STX17 with or without truncated variants of SNX4. Twenty-four hours after transfection, cells were lysed and immunoprecipitated with anti-Flag antibody. Immunoblotting was performed with the indicated antibodies. j, Transmembrane and SNARE domains of STX17 bind to BAR domain of SNX4. HEK293T cells were transfected with empty vector, HA-SNX4 BAR with or without truncated variants of STX17. Twenty-four hours after transfection, cells were lysed and immunoprecipitated with anti-Flag antibody. Immunoblotting was performed with the indicated antibodies. 


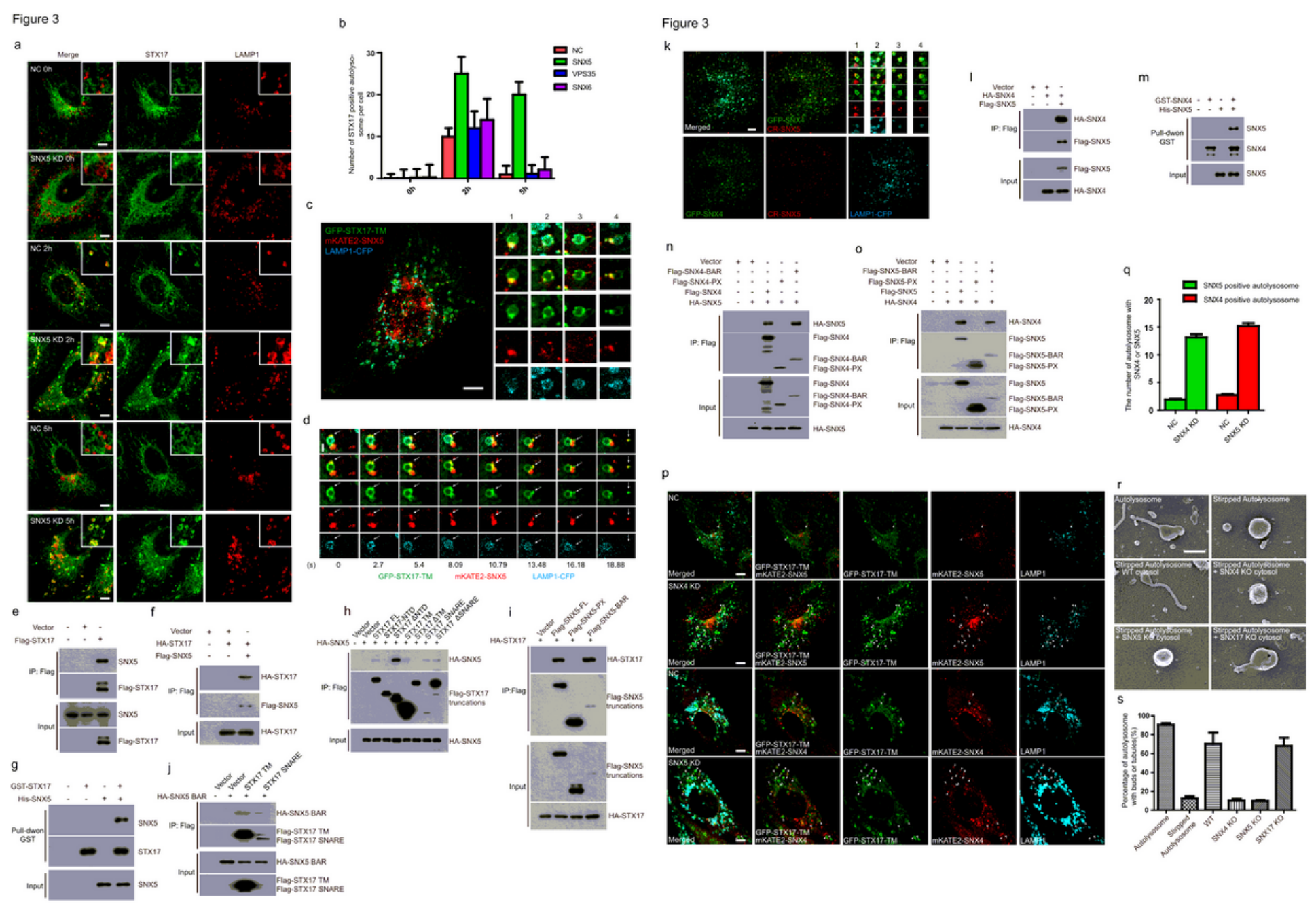

Figure 3

SNX5 cooperates with SNX4 to mediate STX17 sorting from autolysosomes a, SNX5 depletion leads to defects in ACR. U2OS cells stably expressing Flag-STX17 were transfected with non-targeting siRNA (NC) or siRNA against SNX5. Forty-eight hours after transfection, cells were starved with EBSS for the indicated hours. Scale bar, $5 \mu \mathrm{m}$. b, Quantification of STX17-positive autolysosome number. Images in a and extended data figure 3a were analyzed. Data are mean \pm SEM for 100 cells. c, SNX5 localizes to autolysosomes. U2OS cells stably expressing GFP-STX17-TM, mKATE2-SNX5, and LAMP1-CFP were starved with EBSS for $2 \mathrm{~h}$ and live images were taken. Scale bar, $5 \mu \mathrm{m} . \mathrm{d}$, U2OS cells stably expressing GFP-STX17-TM, mKATE2-SNX5, and LAMP1-CFP were starved with EBSS for $2 \mathrm{~h}$ and movies were taken. Scale bar, $1 \mu \mathrm{m}$. e, Endogenous SNX5 interacts with STX17. HEK293T cells were transfected with empty vector or Flag-STX17. Twenty-four hours after transfection, cells were lysed and immunoprecipitated with anti-Flag antibody. Immunoblotting was performed with indicated antibodies. f, SNX5 interacts with STX17. HEK293T cells were transfected with empty vector or HA-STX17 with or without Flag-SNX5. Twenty-four hours after transfection, cells were lysed and immunoprecipitated with anti-Flag antibody. Immunoblotting was performed with the indicated antibodies. g, Direct binding of SNX5 with STX17 in vitro. Glutathione Sepharose beads bound with GST or GST-STX17 were incubated with purified His-SNX5 for $16 \mathrm{~h}$, and then eluted for immunoblotting. $\mathrm{h}$, SNX5 interacts with the transmembrane and SNARE 
domains of STX17. HEK293T cells were transfected with empty vector or HA-SNX5 with or without truncated variants of STX17. Twenty-four hours after transfection, cells were lysed and immunoprecipitated with anti-Flag antibody. Immunoblotting was performed with the indicated antibodies. i, STX17 interacts with the BAR domain of SNX5. HEK293T cells were transfected with empty vector or HA-STX17 with or without truncated variants of SNX5. Twenty-four hours after transfection, cells were lysed and immunoprecipitated with anti-Flag antibody . j, Transmembrane and SNARE domains of STX17 bind to BAR domain of SNX5. HEK293T cells were transfected with empty vector, HASNX5 BAR with or without truncated variants of STX17. Twenty-four hours after transfection, cells were lysed and immunoprecipitated with anti-Flag antibody. Immunoblotting was performed with the indicated antibodies. k, SNX4 and SNX5 co-localize to lysosomes. Hela cells were transfected with GFP-SNX4, mCherry-SNX5, and LAMP1-CFP. Scale bar, $5 \mu \mathrm{m}$. I, SNX4 interacts with SNX5 in vivo. HEK293T cells were transfected with empty vector or Flag-SNX5 with or without HA-SNX4. Twenty-four hours after transfection, cells were lysed and immunoprecipitated with anti-Flag antibody. Immunoblotting was performed with the indicated antibodies. $m$, Direct binding of SNX4 with SNX5 in vitro. Glutathione Sepharose beads bound with GST or GST-SNX4 were incubated with or without purified His-SNX5 for 16h, and then eluted for immunoblotting. $n$, SNX5 interacts with the BAR domain of SNX4. HEK293T cells were transfected with HA-SNX5 with empty vector, Flag-SNX4, Flag-SNX4-BAR, or Flag-SNX4-PX. Twenty-four hours after transfection, cells were lysed and immunoprecipitated with anti-Flag antibody. Immunoblotting was performed with the indicated antibodies. O, SNX4 interacts with the BAR domain of SNX5. HEK293T cells were transfected with HA-SNX4 with empty vector and Flag-SNX5, Flag-SNX5-BAR, or Flag-SNX5-PX. Twenty-four hours after transfection, cells were lysed and immunoprecipitated with anti-Flag antibody. Immunoblotting was performed with the indicated antibodies. p, SNX4 and SNX5 depletion block the disassociation of each other from autolysosomes. U2OS cells stably expressing GFPSTX17-TM, LAMP1-CFP, and mKATE2-SNX5 or mKATE2-SNX4 were transfected with non-targeting siRNA (NC) or siRNA against SNX4 or SNX5. Forty-eight hours after transfection, cells are starved with EBSS for 2 hours. Arrows indicate the autolysosomes with SNX4 or SNX5. Scale bar, $5 \mu \mathrm{m}$. q, Quantification of SNX4 or SNX5 localization to autolysosomes in p. The number of autolysosomes with SNX4 or SNX5 was quantified. Data are means \pm SEM for 100 cells. r, Both SNX4 and SNX5 are required for generating membrane curvature on autolysosomes. Purified autolysosomes from starved MEF cells were treated with trypsin, incubated with the indicated cytosol, washed and then observed under scanning electron microscopy. s, Samples from $r$ were quantified to determine the percentage of autolysosome with buds or tubules. Data are means \pm SEM. $n=50$ autolysosomes from at least three independent experiments. 


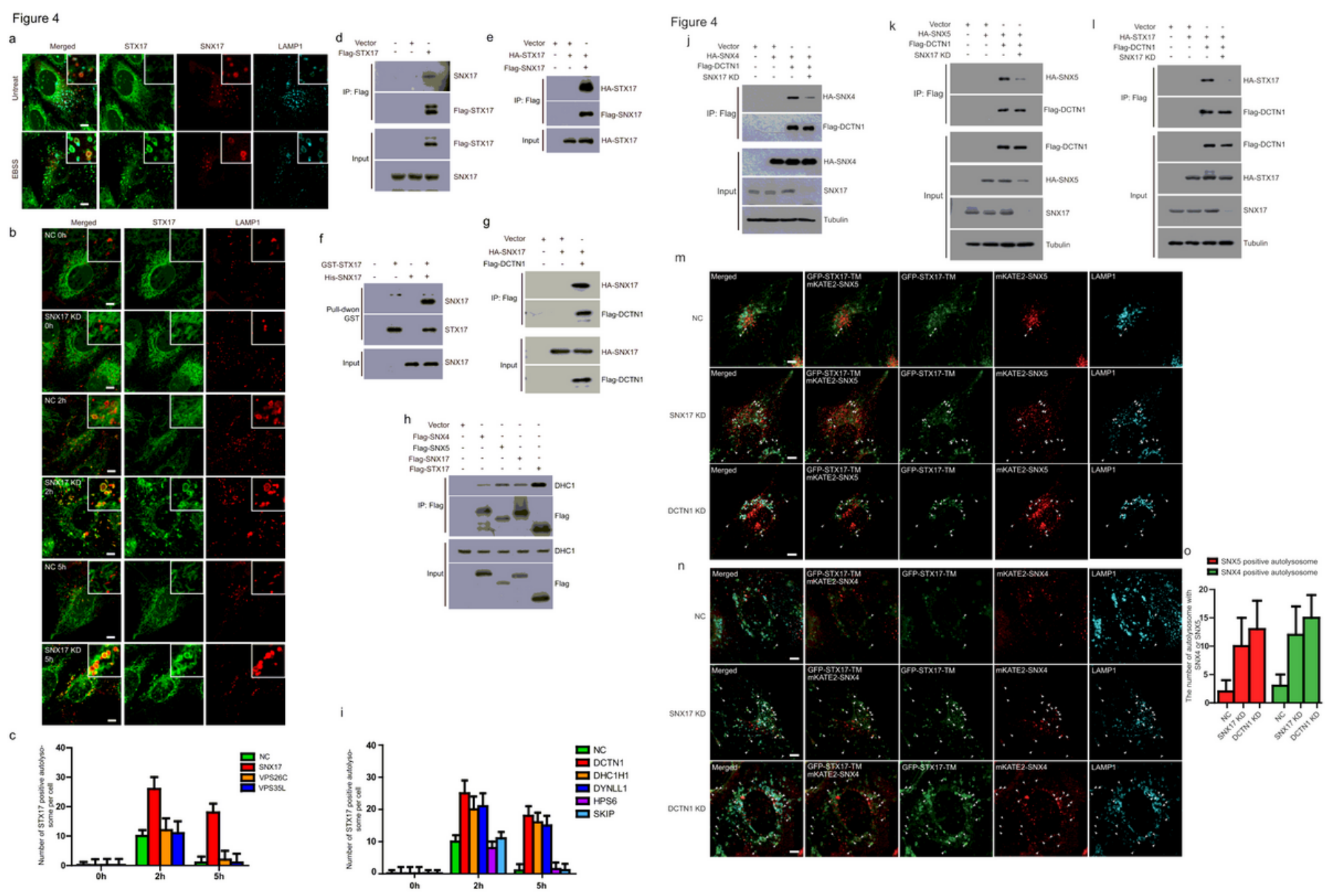

Figure 4

SNX17 interacts with both the STX17-SNX4-SNX5 and dynein-dynactin complexes to facilitate STX17 retrieval a, SNX17 localizes to autolysosomes. U20S cells stably expressing GFP-STX17-TM mKATE2SNX17 and LAMP1-CFP were starved with EBSS for 2 hours. Scale bar, $5 \mu \mathrm{m}$. b, SNX17 is required for ACR. U20S cells stably expressing Flag-STX17 were transfected with non-targeting siRNA (NC) or siRNA against SNX17. Forty-eight hours after transfection, cells were starved with EBSS for the indicated hours. Scale bar, $5 \mu \mathrm{m}$. c, Quantification of STX17-positive autolysosome number. Images in b and Extended Data Fig4a were analyzed. Data are means \pm SEM for 100 cells. d, Endogenous SNX17 interacts with STX17. HEK293T cells were transfected with empty vector or Flag-STX17. Twenty-four hours after transfection, cells were lysed and immunoprecipitated with anti-Flag antibody. Immunoblotting was performed with the indicated antibodies. e, SNX17 interacts with STX17. HEK293T cells were transfected with empty vector or Flag-SNX17 with or without HA-STX17. Twenty-four hours after transfection, cells were lysed and immunoprecipitated with anti-Flag antibody. Immunoblotting was performed with the indicated antibodies. f, Direct binding of SNX17 with STX17 in vitro. Glutathione Sepharose beads bound with GST or GST-STX17 were incubated with purified His-SNX17 for 16h, and then eluted for immunoblotting. g, SNX17 interacts with DCTN1 in vivo. HEK293T cells were transfected with empty vector or HA-SNX17 with or without Flag-DCTN1. Twenty-four hours after transfection, cells were lysed 
and immunoprecipitated with anti-Flag antibody. Immunoblotting was performed with the indicated antibodies. h, SNX17 interacts with DHC1. HEK293T cells were transfected with empty vector, FlagSNX17, Flag-SNX4, Flag-SNX5, and Flag-STX17. Twenty-four hours after transfection, cells were lysed and immunoprecipitated with anti-Flag antibody. Immunoblotting was performed with the indicated antibodies. i, Quantification of STX17-positive autolysosome number. Images in Extended Data Fig. 5b and Extended Data Fig. 6a were analyzed. Data are means \pm SEM for 100 cells. j-I, SNX17 depletion leads to reduced interactions of DCTN1 with SNX4, SNX5, and STX17. HEK293T cells were transfected with non-targeting siRNA or siRNA against SNX17. Twenty-four hours after transfection, cells were transfected with Flag- DCTN1 with HA-SNX4, HA-SNX5, or HA-STX17. Another twenty-four hours after transfection, cells were lysed and immunoprecipitated with anti-Flag antibody. Immunoblotting was performed with the indicated antibodies. m-n, U2OS cells stably expressing GFP-STX17-TM, mKATE2-SNX5, and LAMP1CFP or cells stably expressing GFP-STX17-TM, mKATE2-SNX4, and LAMP1-CFP were transfected with non-targeting siRNA (NC), siRNA against SNX17, or DCTN1. Forty-eight hours after transfection cells were starved with EBSS for 2 hours. Arrows indicate the autolysosomes with SNX4 or SNX5. Scale bar, 5um. o, Quantification of SNX4- or SNX5-positive autolysosomes in $\mathrm{m}$ and $\mathrm{n}$. The number of autolysosomes with SNX4 or SNX5 was quantified. Data are means \pm SEM for 100 cells. 
a

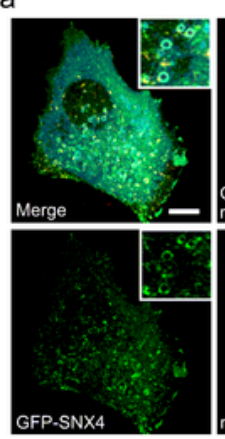

C

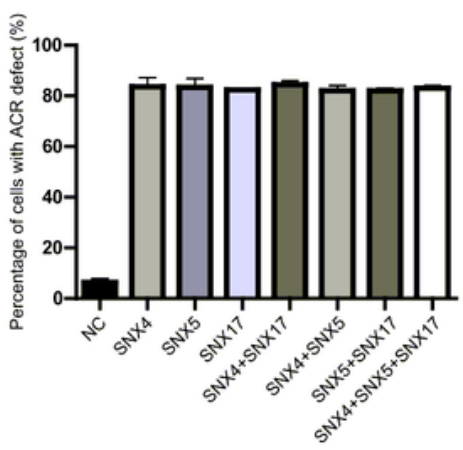

e

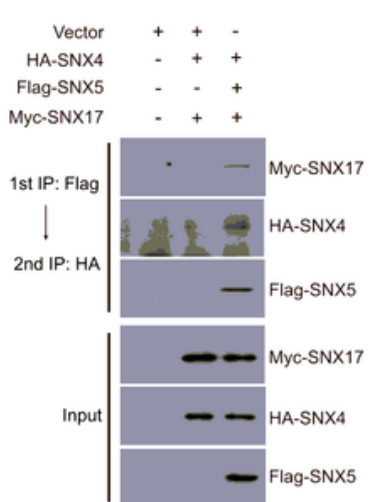

g

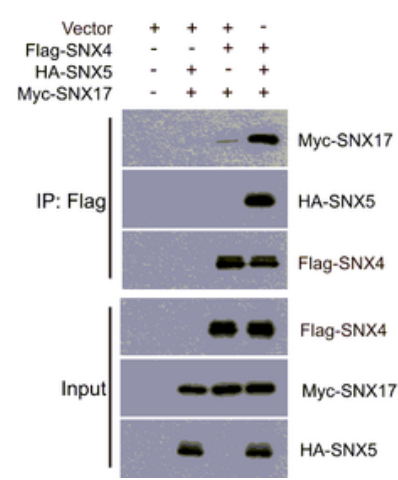

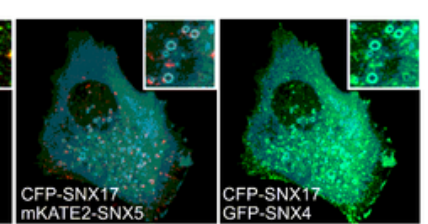

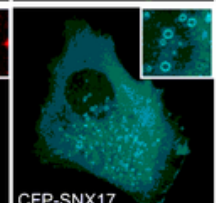

d
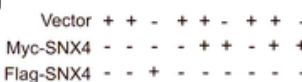

HA-SNX5 $+++\cdots++$

Flag-SNX5 - . . + . -

Myc-SNX17 - + + . . -

HA-SNX17 - - - + + -
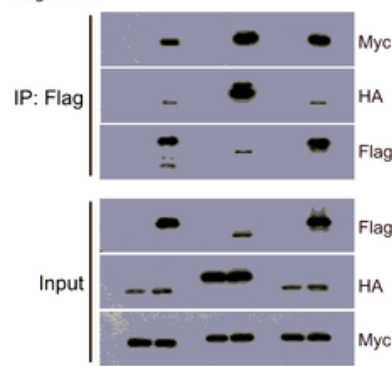

$f$

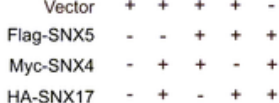

HA-SNX17

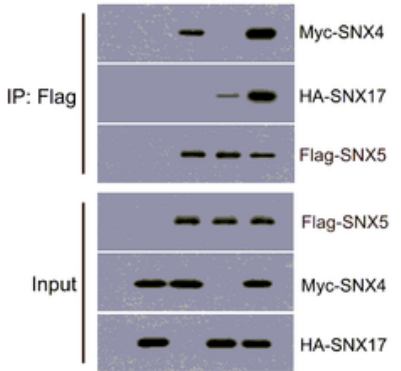

h
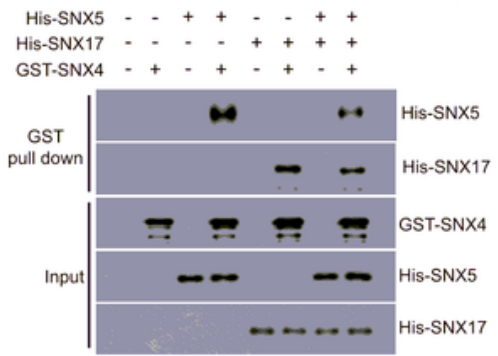
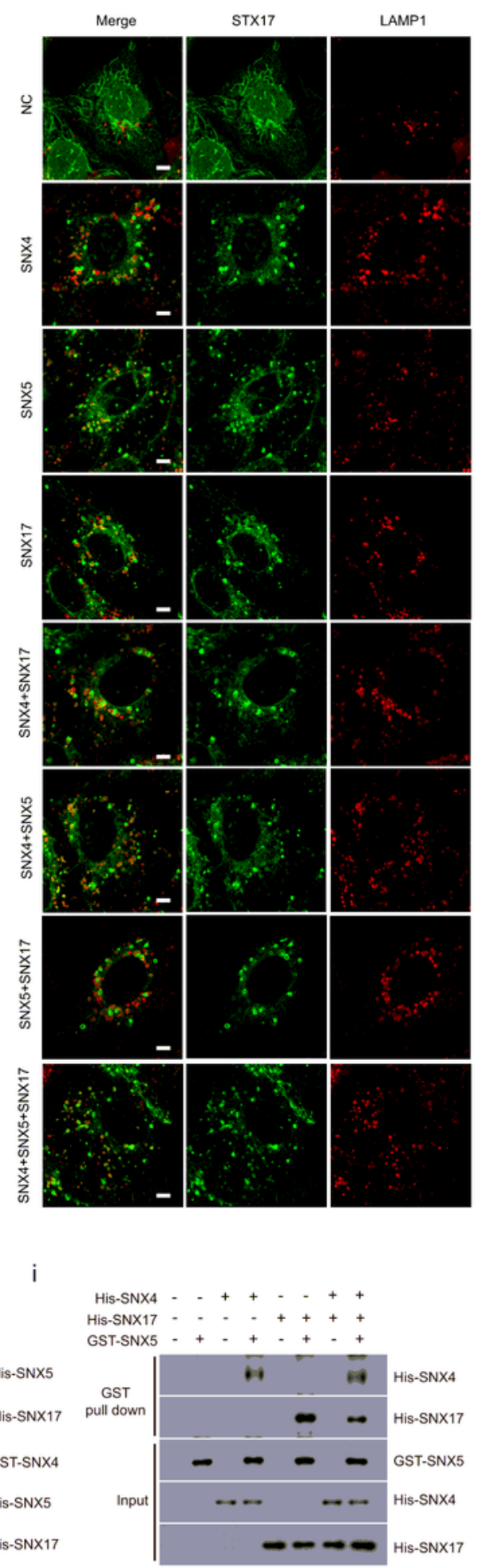

Figure 5

SNX4-SNX5-SNX17 form the recycler complex a, SNX4, SNX5, and SNX17 co-localize with each other.

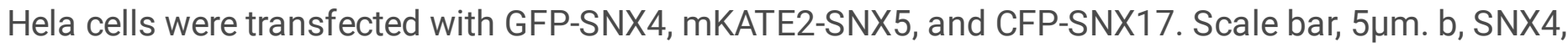
SNX5, and SNX17 function in the same pathway. U2OS cells stably expressing GFP-STX17-TM and LAMP1-mCherry were transfected with non-targeting siRNA (NC) or siRNA against SNX4, SNX5, and SNX17. Forty-eight hours after transfection cells were starved with EBSS for 5 hours. Scale bar, $5 \mu \mathrm{m}$. C, 
Quantification of cells with defects in ACR in b. Data are means \pm SEM for 50 cells. d, SNX4, SNX5, and SNX17 interact with each other. 293T cells were transfected with plasmids as indicated. Twenty-four hours after transfection, cells were lysed and immunoprecipitated with anti-Flag antibody. Immunoblotting was performed with the indicated antibodies. e, SNX4, SNX5, and SNX17 form a complex. HEK293T cells were transfected with HA-SNX4, Flag-SNX5, and Myc-SNX17. Twenty-four hours after transfection, cells were lysed and immunoprecipitated with anti-Flag antibody. The elution from the first immunoprecipitation was subjected to a second round of immunoprecipitation with anti-HA antibody. Immunoblotting was then performed with the indicated antibodies. $f-g$, Enhanced interactions among SNX4, SNX5, and SNX17. HEK293T cells were transfected with the indicated plasmids. Twentyfour hours after transfection, cells were lysed and immunoprecipitated with anti-Flag antibody. h-i, SNX4, SNX5, and SNX17 form a complex in vitro. Purified proteins were incubated together as indicated with GST-beads. After $16 \mathrm{~h}$ of incubation, the elution was subjected to immunoblotting. 
Extended Data Figure 1

a
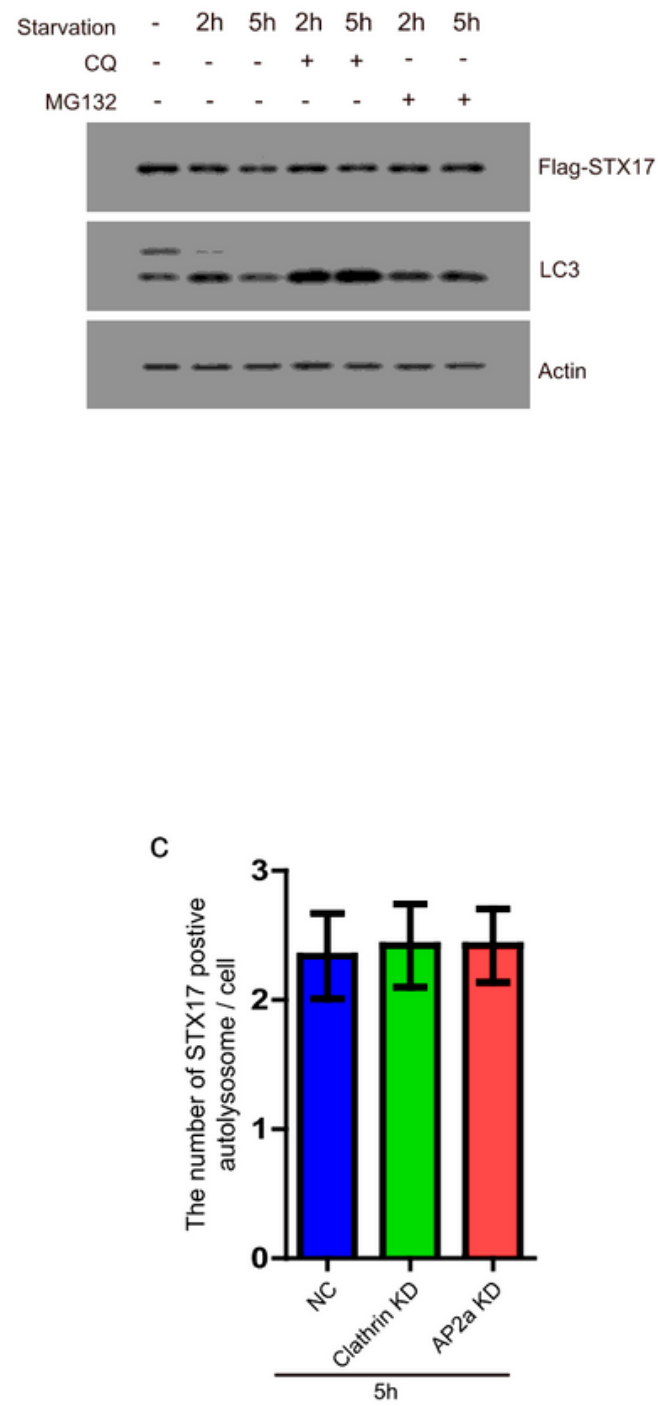

b
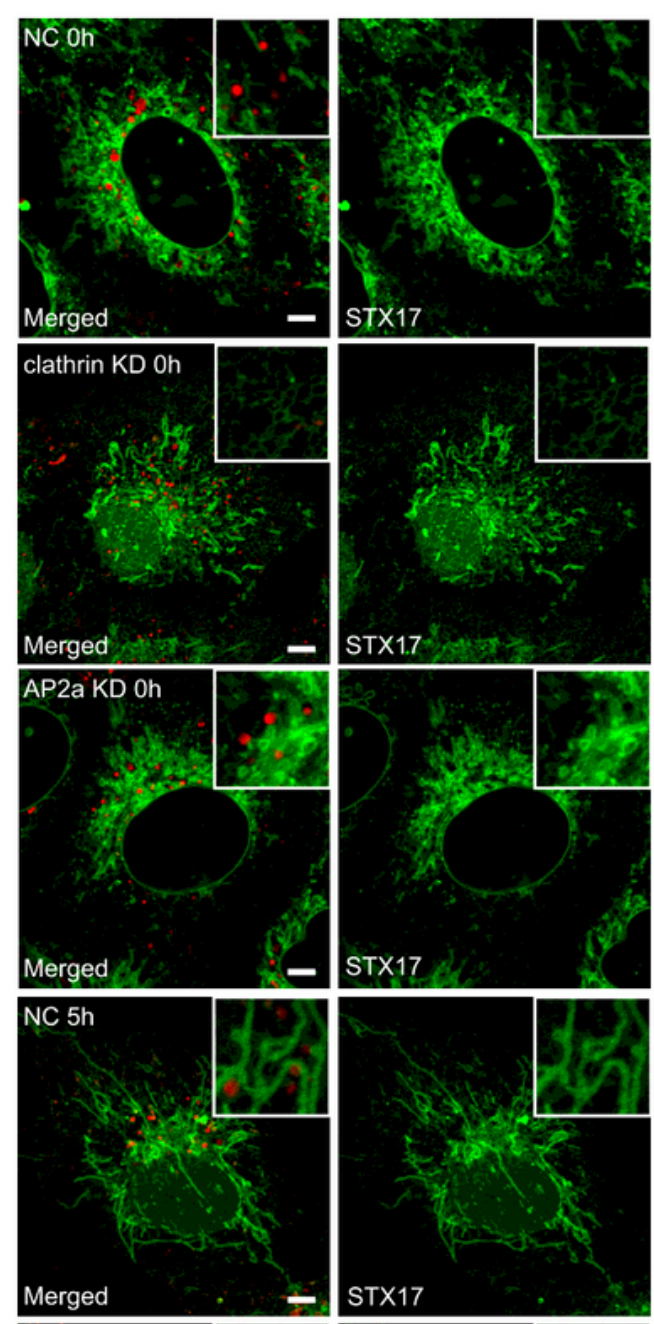

\section{sixiz:}

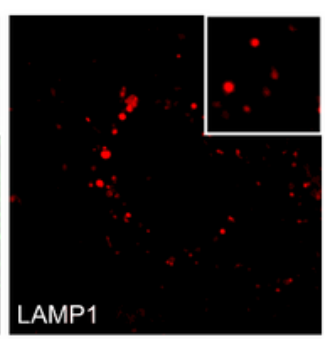

LAMP1
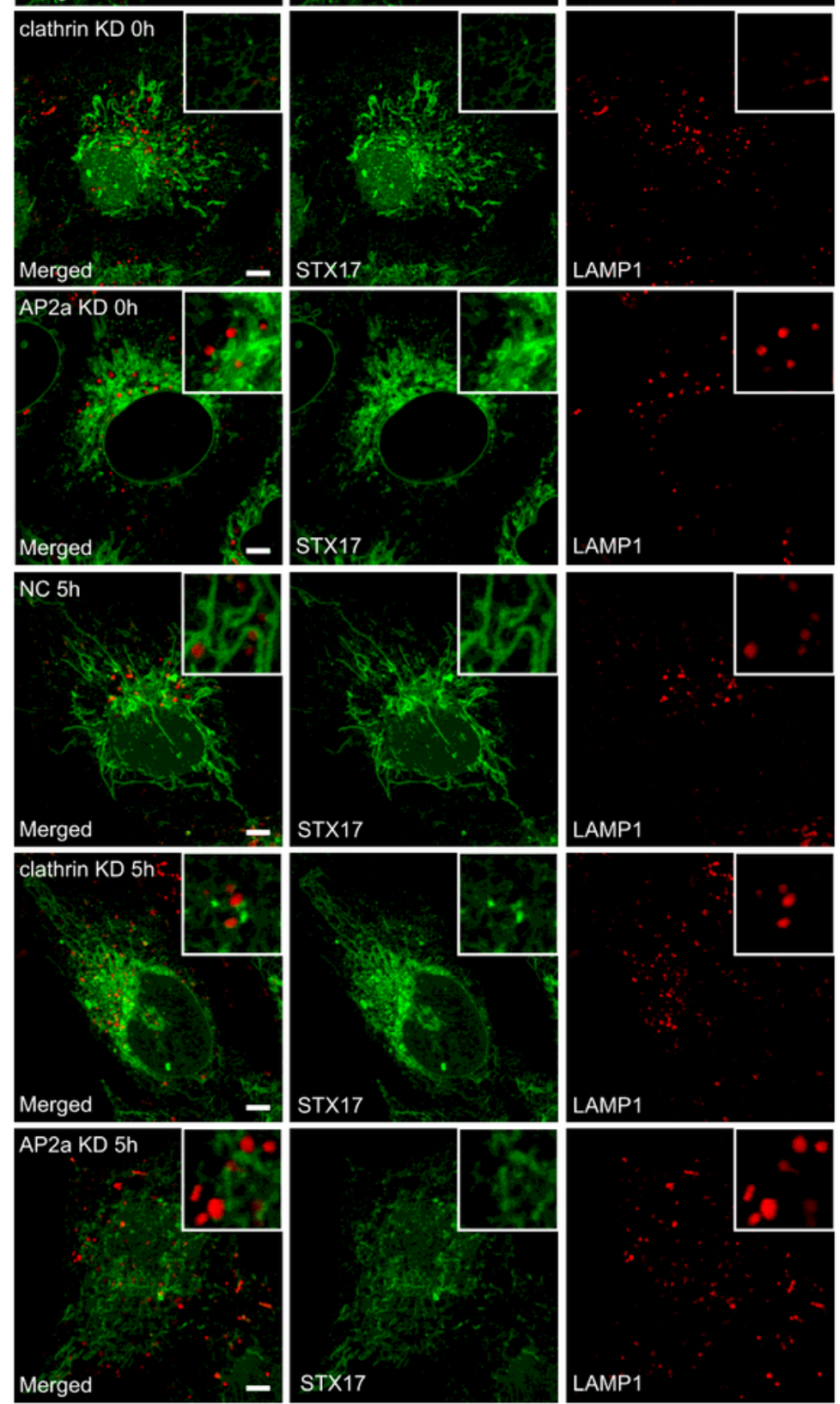

LAMP1
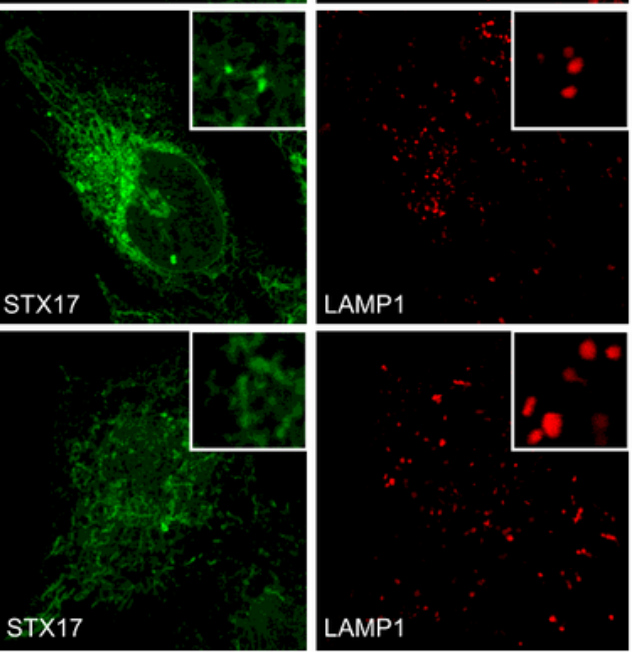

\section{Figure 6}

ALR gene is not required for ACR a, STX17 expression level remains largely unchanged after starvation. U2OS cells stably expressing Flag-STX17 were starved for the indicated hours. Cells were collected and lysed for immunoblotting with the indicated antibodies. b, ALR genes are not required for ACR. U2OS cells stably expressing Flag-STX17 were transfected with the indicated siRNA. Forty-eight hours after 
transfection, cells were starved with EBSS for the indicated hours. Scale bar, $5 \mu \mathrm{m}$. c, Quantification of STX17-positive autolysosomes in b. Data are means \pm SEM for 50 cells.

Extended Data Figure 2

a

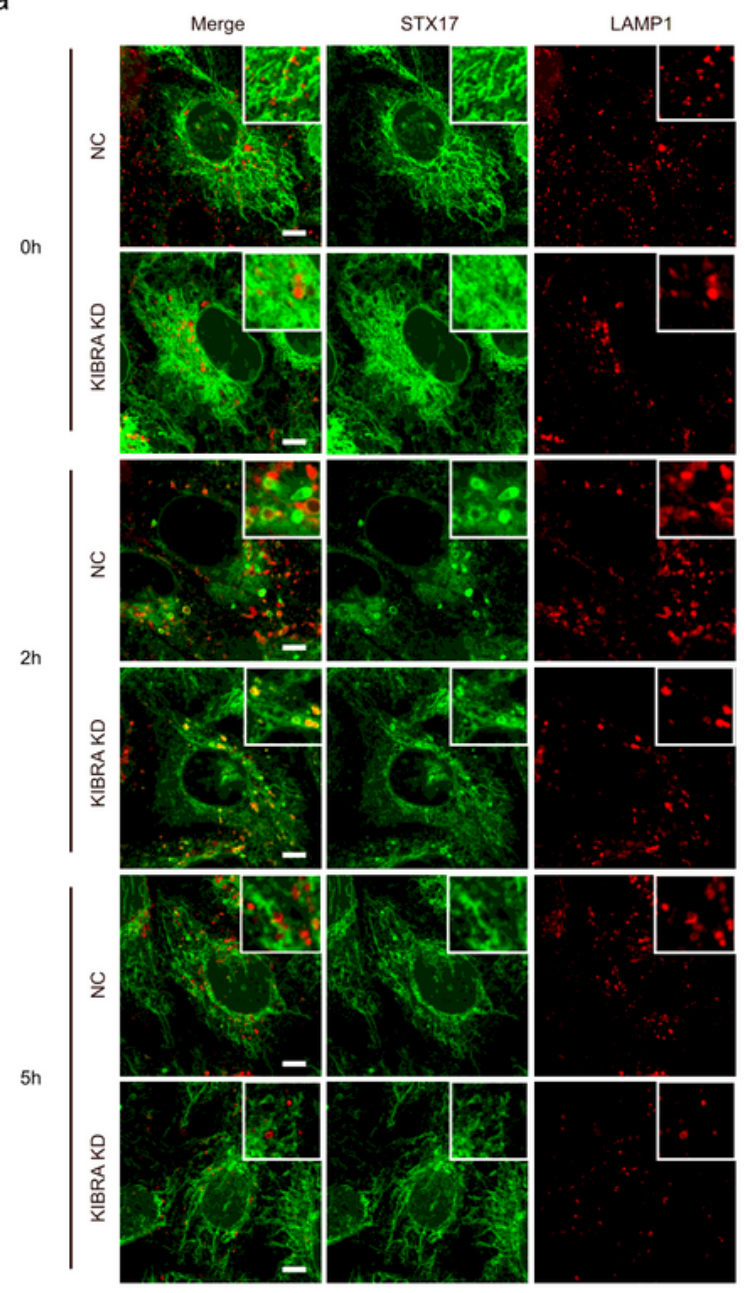

b

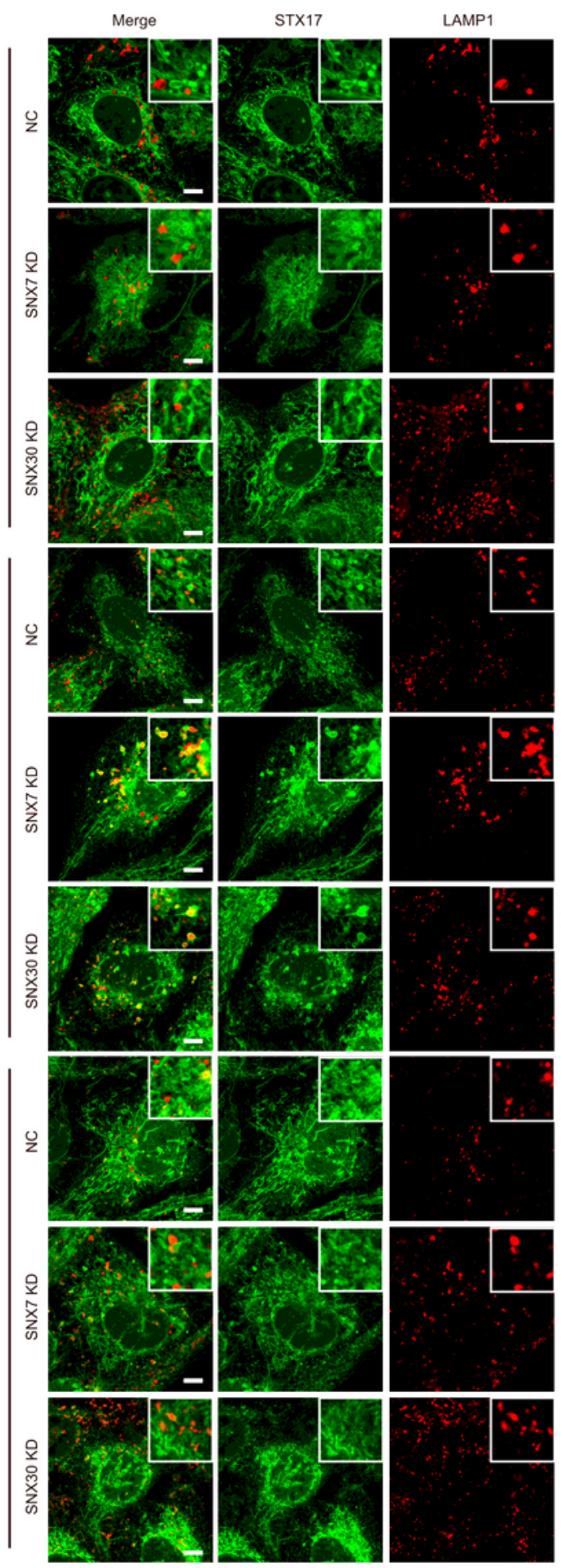

\section{Figure 7}

KIBRA, SNX7, and SNX30 are not required for ACR a-b, KIBRA, SNX7, and SNX30 depletion have no effect on ACR. U2OS cells stably expressing Flag-STX17 were transfected with non-targeting siRNA (NC) or 
siRNA against KIBRA, SNX7, and SNX30. Forty-eight hours after transfection cells were starved with EBSS for the indicated hours. Scale bar, $5 \mu \mathrm{m}$.

\section{Extended Data Figure 3}

a

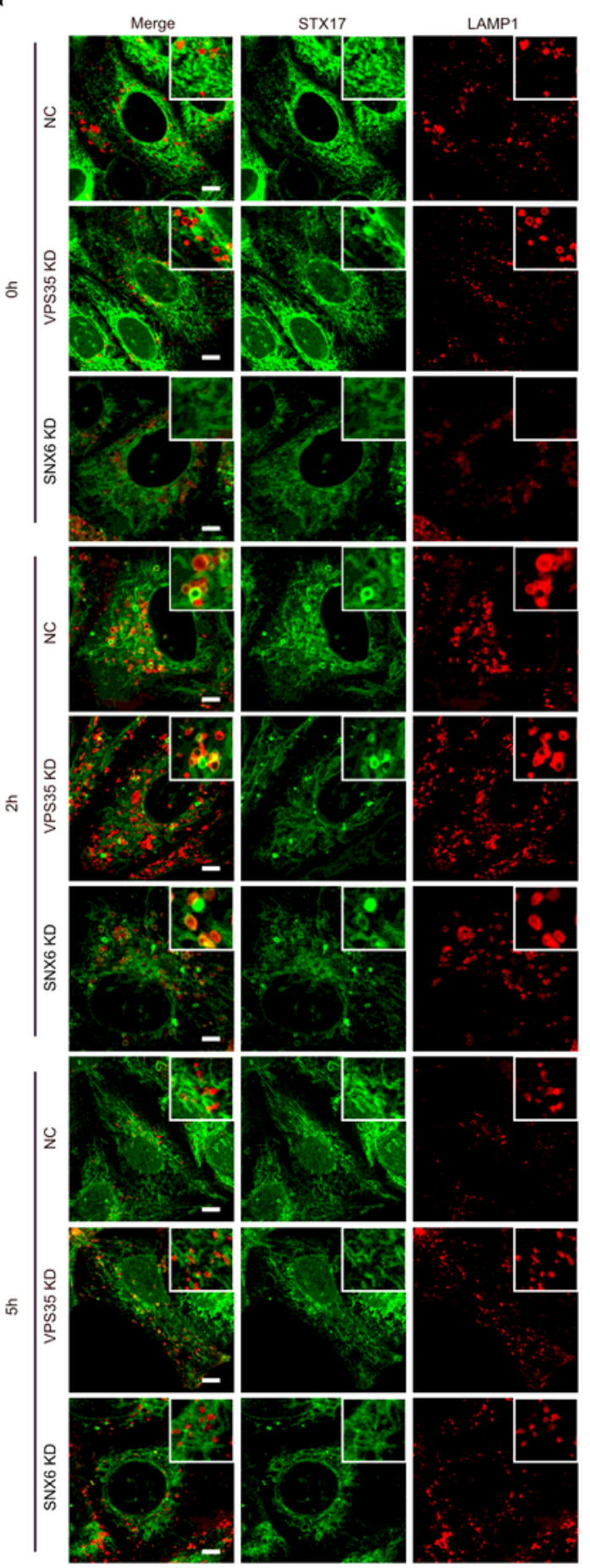

\section{Figure 8}

Retromer is not required for ACR a, VPS35 and SNX6 depletion have no effect on ACR. U2OS cells stably expressing Flag-STX17 were transfected with non-targeting siRNA (NC) or siRNA against VPS35 and 
SNX6. Forty-eight hours after transfection cells were starved with EBSS for the indicated hours. Scale bar, $5 \mu \mathrm{m}$.

Extended Data Figure 4

a
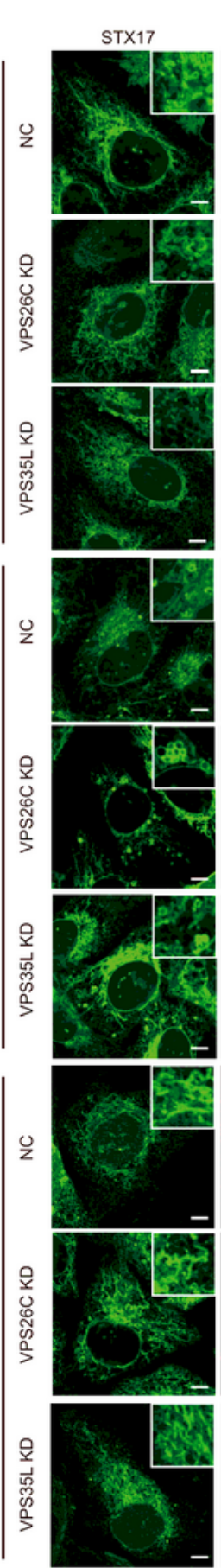

$2 \mathrm{~h}$
LAMP1
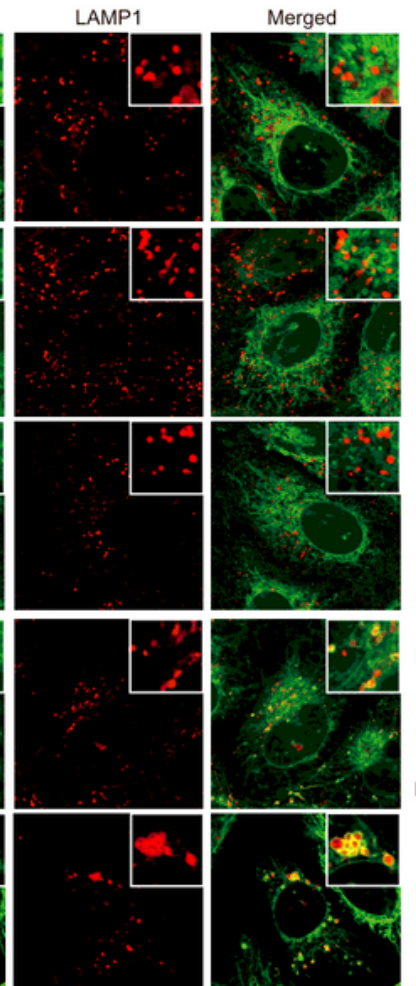

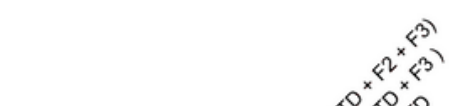

b
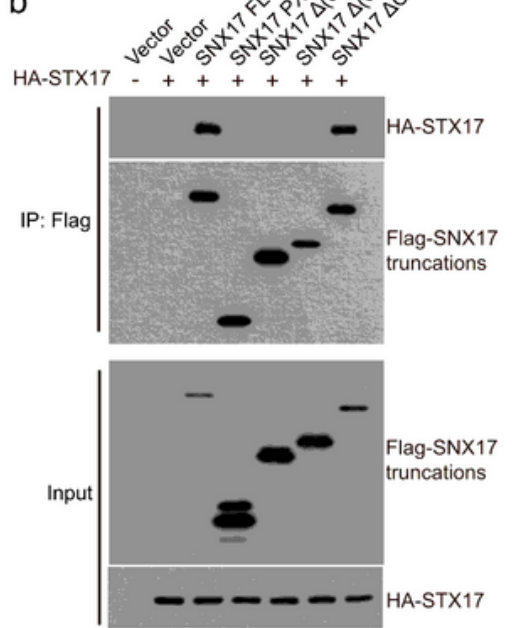

$\mathrm{C}$
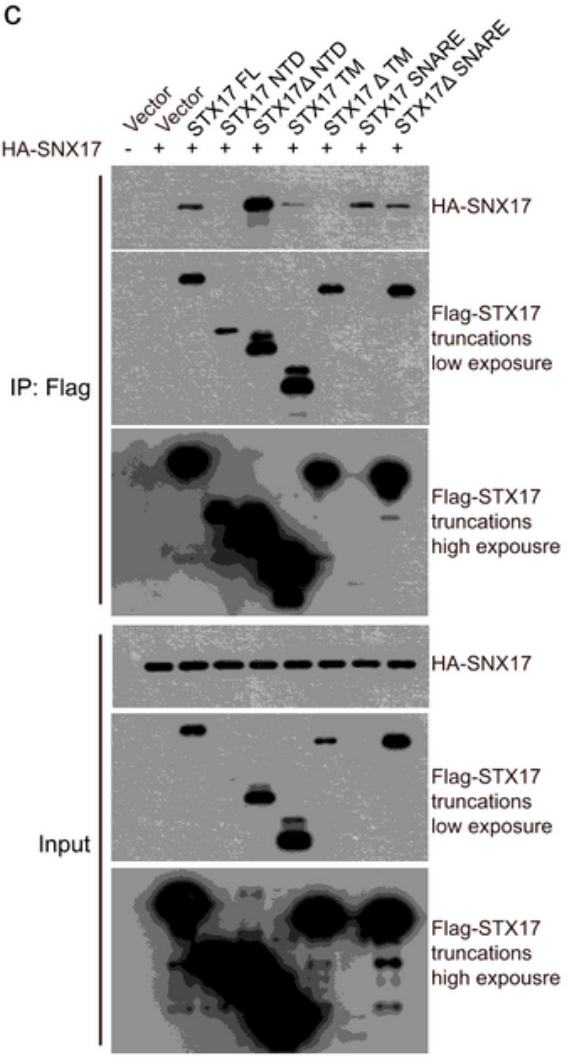
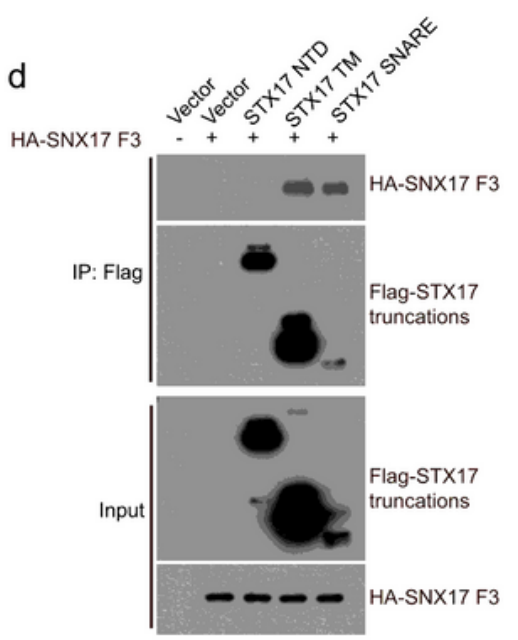
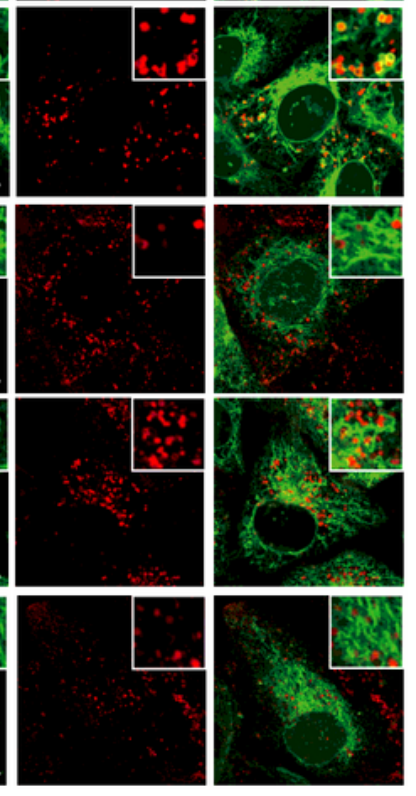

\section{Figure 9}

VPS26C and VPS35L are not required for ACR, and mapping the interaction regions between SNX17 and STX17 a, VPS26C and VPS35L depletion leads to ACR defect. U20S cells stably expressing Flag-STX17 were transfected with non-targeting siRNA (NC) or siRNA against VPS26C or VPS35L. Forty-eight hours 
after transfection cells were starved with EBSS for the indicated hours. Scale bar, $5 \mu \mathrm{m}$. b-d, The transmembrane, and SNARE domains of STX17 interact with the F3 region in the FERM domain of SNX17. HEK293T cells were transfected with the indicated plasmids. Twenty-four hours after transfection, cells were lysed and immunoprecipitated with anti-Flag antibody. Immunoblotting was performed with the indicated antibodies.

\section{Extended Data Figure 5}

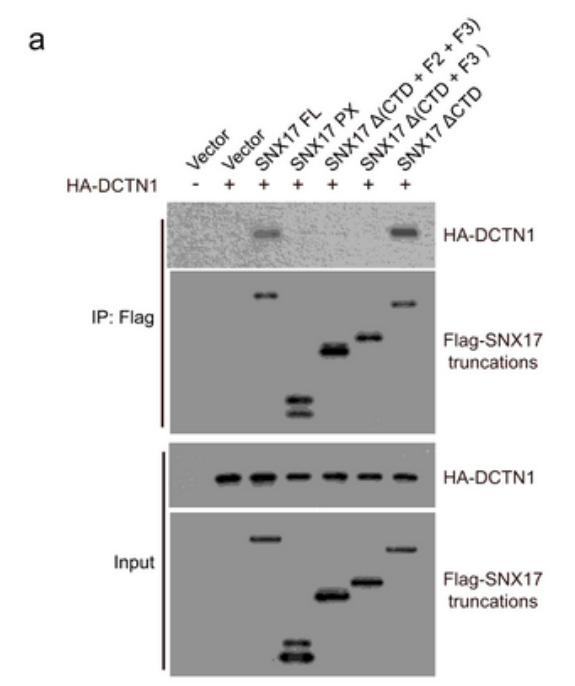

b
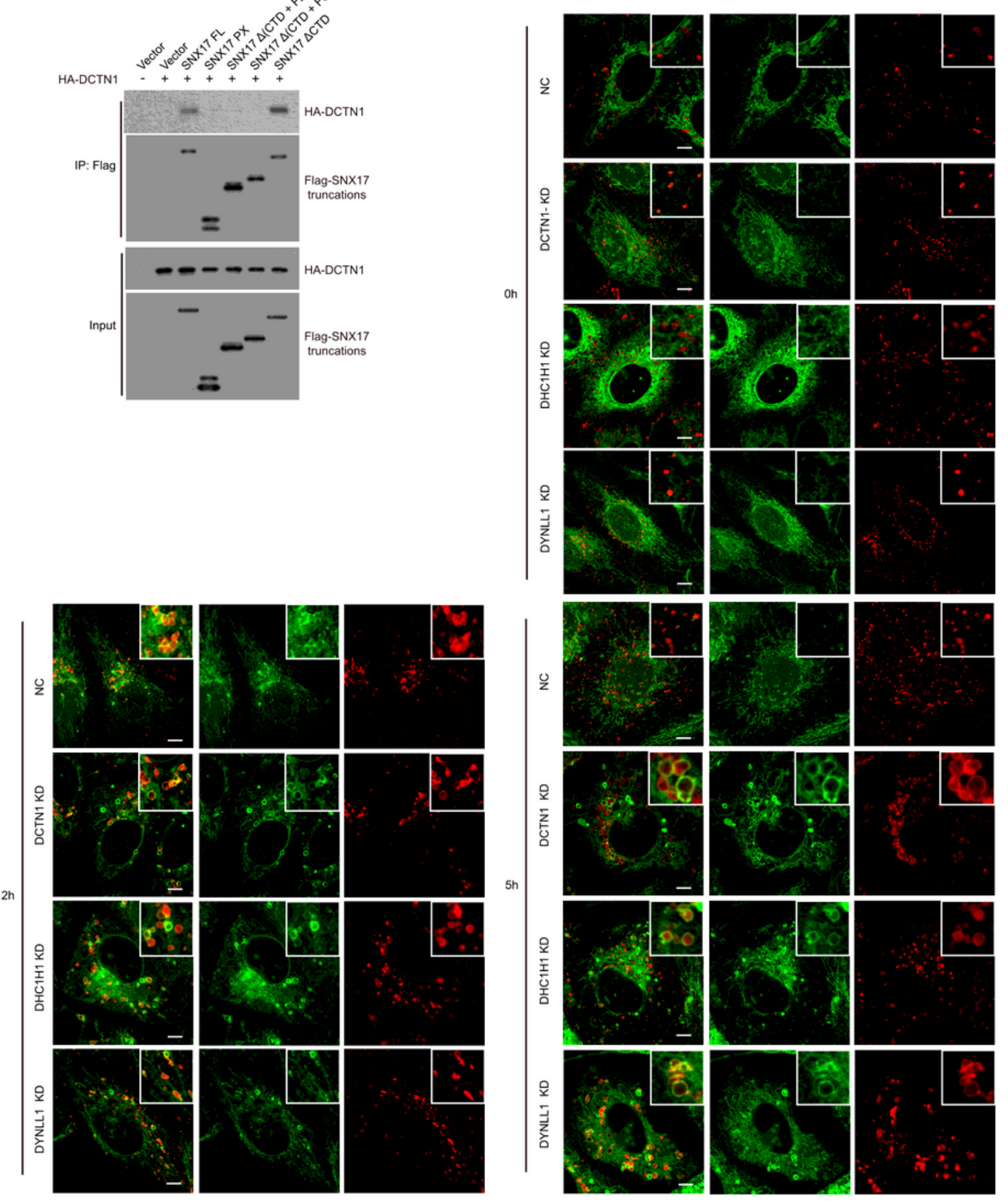

Figure 10 
Dynein-dynactin complex is required for ACR a, DCTN1 interacts with the F3 region in the FERM domain of SNX17. HEK293T cells were transfected with empty vector or HA-DCTN1 with or without truncated variants of SNX17. Twenty-four hours after transfection, cells were lysed and immunoprecipitated with anti-Flag antibody. b, Depletion of subunits of the dynein-dynactin complex leads to ACR defect. U2OS cells stably expressing Flag-STX17 were transfected with non-targeting siRNA (NC) or siRNA against DCTN1, DHC1H1, or DYNLL1. Forty-eight hours after transfection cells were starved with EBSS for the indicated hours. Scale bar, $5 \mu \mathrm{m}$.

Extended Data Figure 6

a

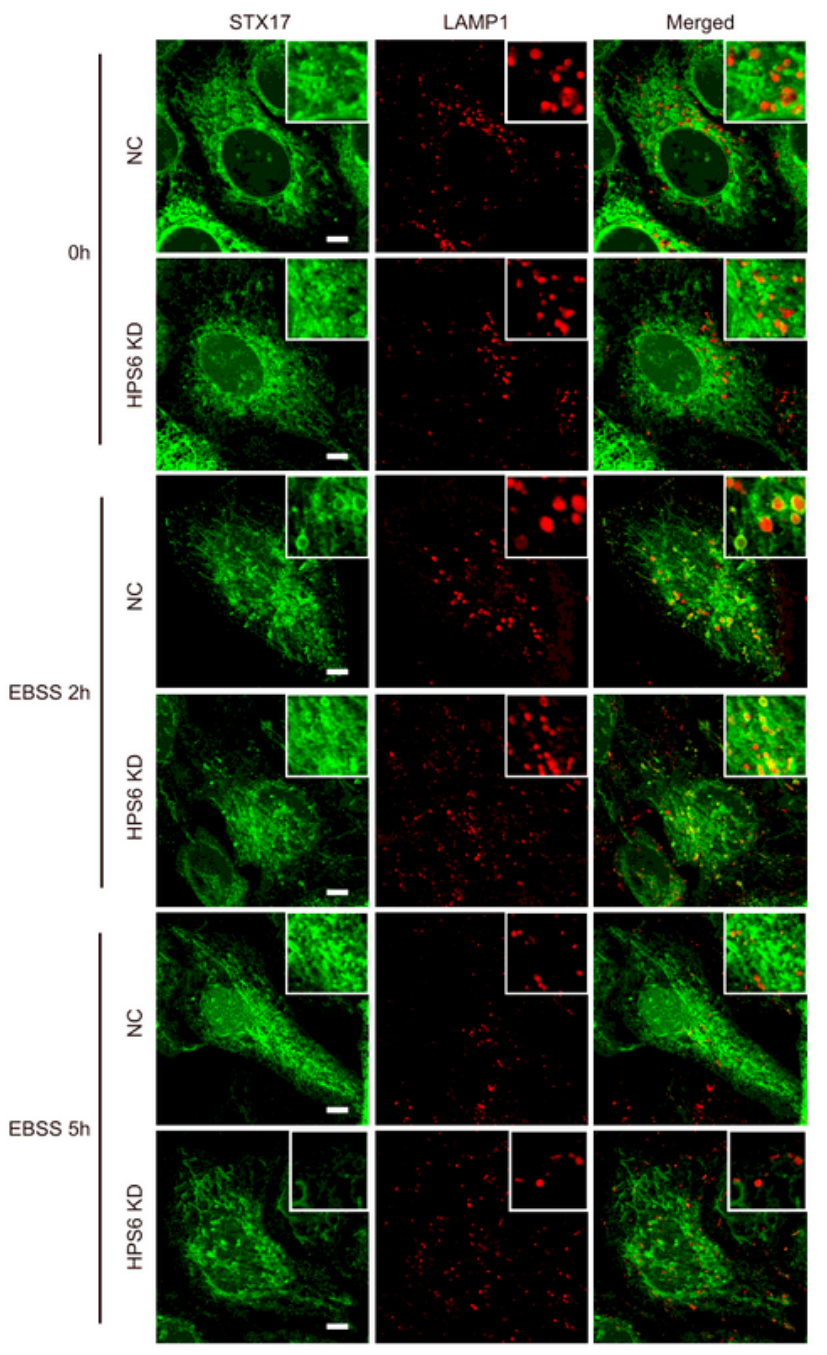

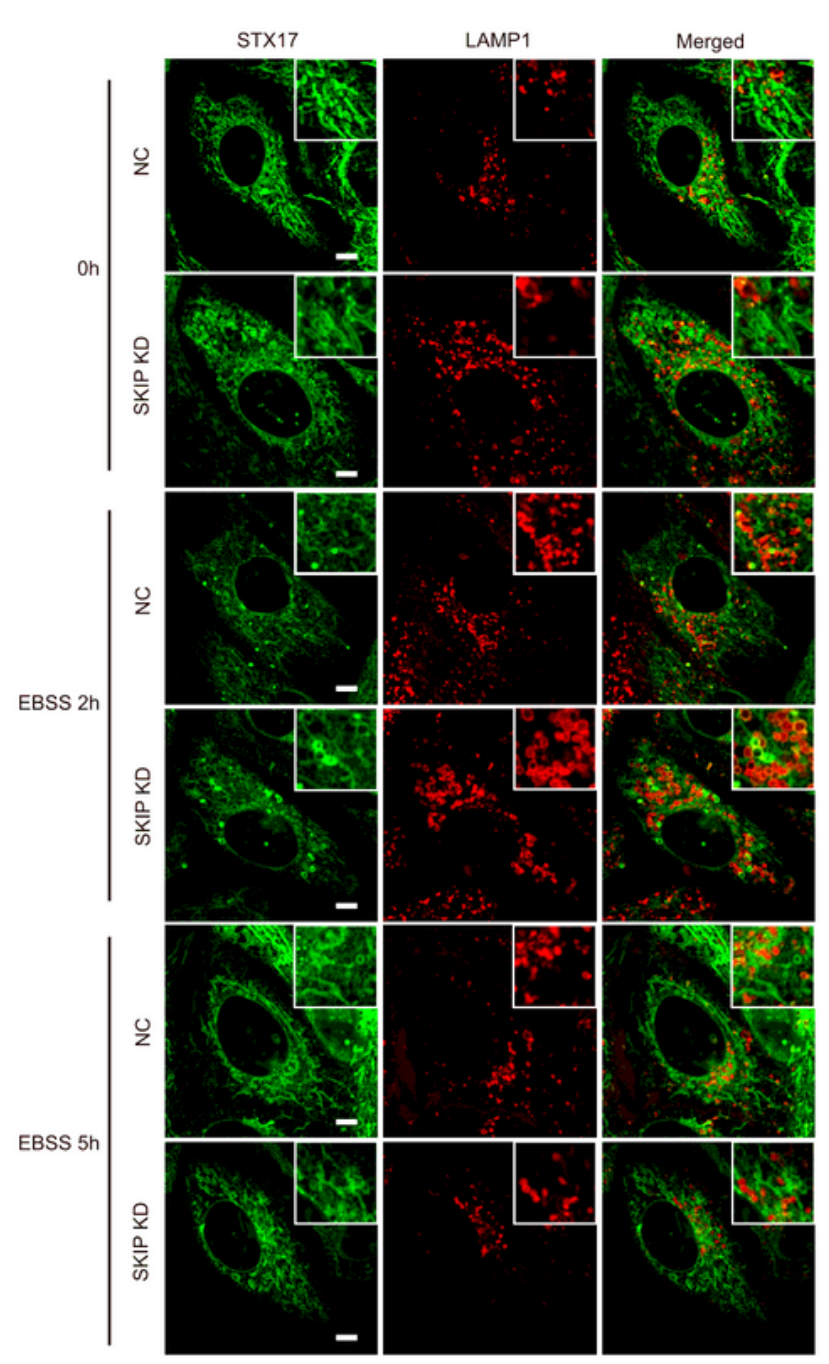

d

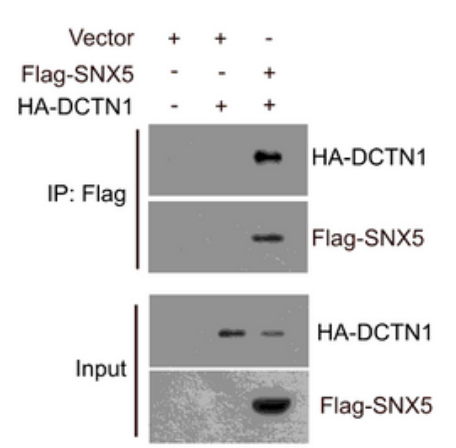

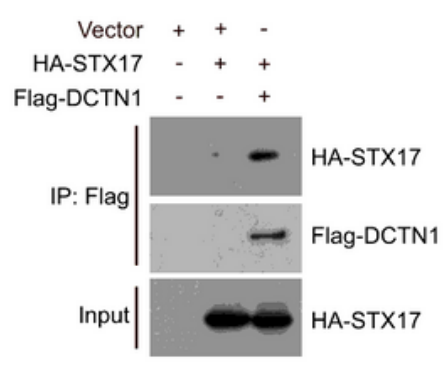




\section{Figure 11}

HPS6 and SKIP are not required for ACR a, HPS6 and SKIP depletion leads to ACR defect. U2OS cells stably expressing Flag-STX17 were transfected with non-targeting siRNA (NC) or siRNA against HPS6 or SKIP. Forty-eight hours after transfection cells were starved with EBSS for the indicated hours. Scale bar, $5 \mu \mathrm{m}$. b-d, DCTN1 interacts with SNX4, SNX5, and STX17. HEK293T cells were transfected with the indicated plasmids. Twenty-four hours after transfection, cells were lysed and immunoprecipitated with anti-Flag antibody. Immunoblotting was performed with the indicated antibodies.

Extended Data Figure 7

a

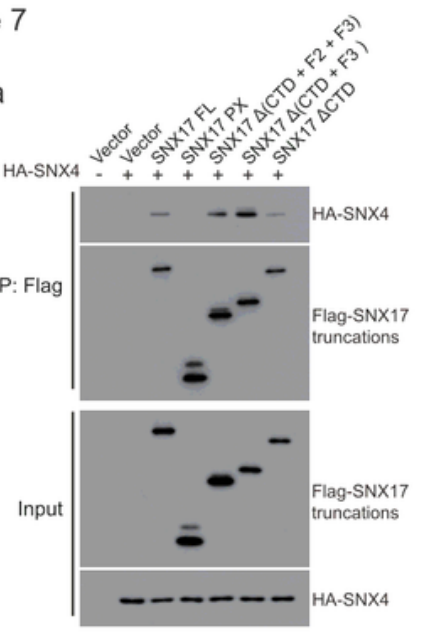

$b$

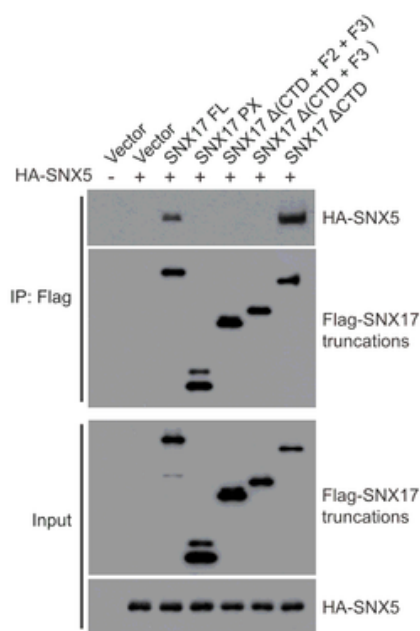

e
C
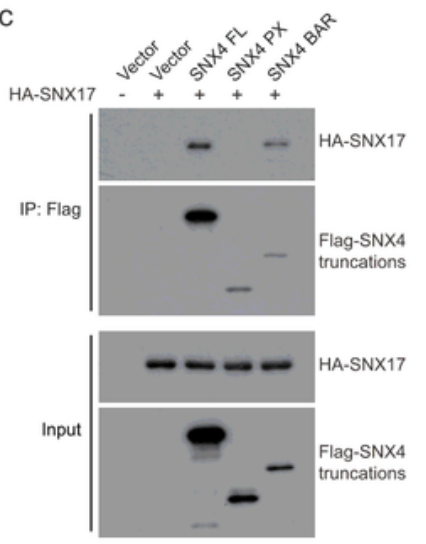

f

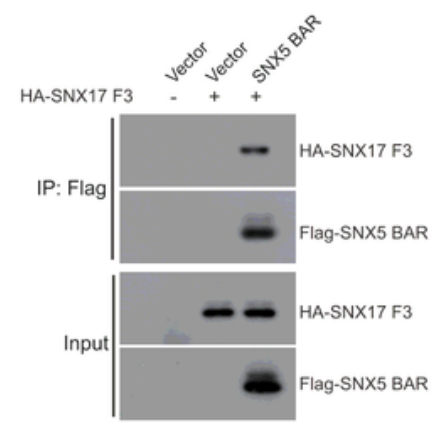

h d

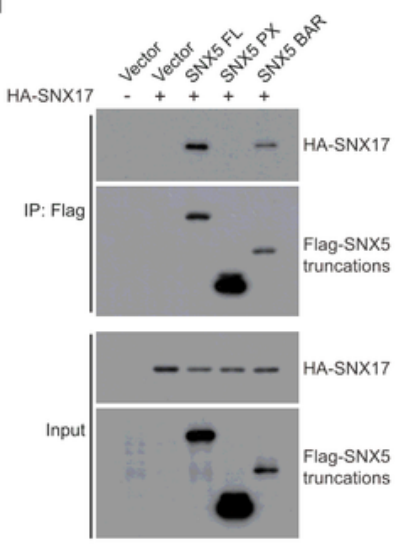

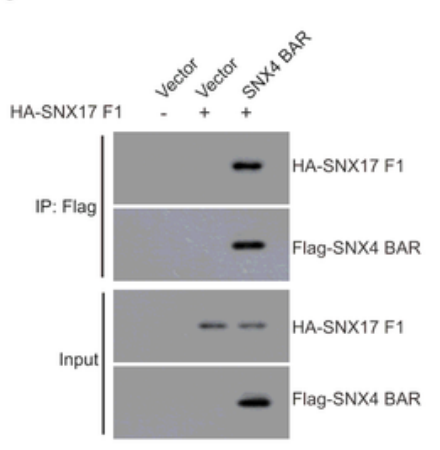

g

$\begin{array}{llllllllllllllll}\operatorname{EBSS}(\mathrm{h}) & - & 2 & 5 & - & - & 2 & 5 & - & 2 & 5 & - & - & 2 & 5 \\ \mathrm{CQ}(\mathrm{h}) & - & - & - & 2 & 5 & 2 & 5 & - & - & - & 2 & 5 & 2 & 5\end{array}$

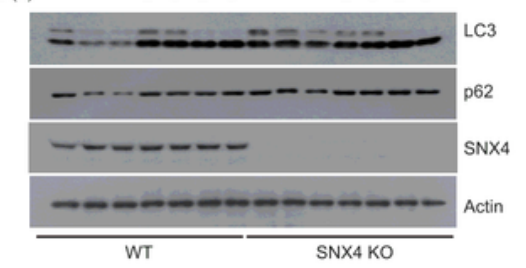

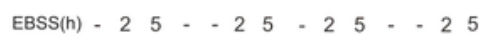
$\mathrm{CQ}(\mathrm{h})-\cdots \quad 2525 \cdots-2525$
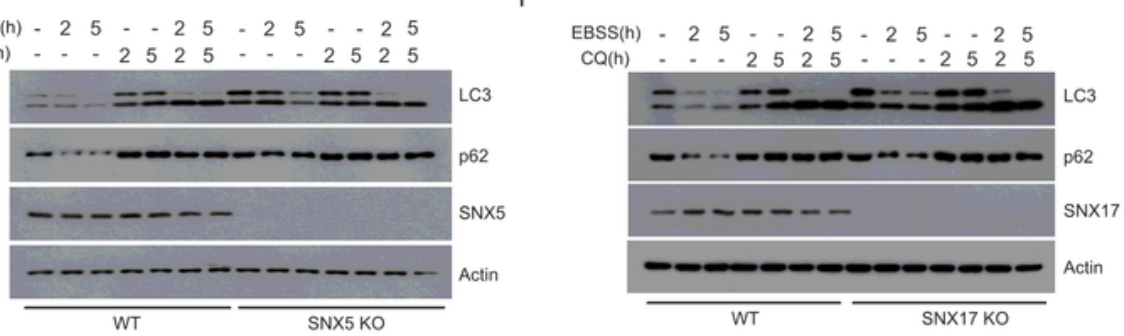

Page 37/39 
Figure 12

The binding regions among SNX4, SNX5, and SNX17 a-f, SNX17 interacts with the BAR domains of SNX4 and SNX5 through its F1 and F3 regions, respectively. HEK293T cells were transfected with the indicated plasmids. Twenty-four hours after transfection, cells were lysed and immunoprecipitated with anti-Flag antibody. Immunoblotting was performed with the indicated antibodies. g-i, Autophagic flux was inhibited in SNX4 KO, SNX5 KO, or SNX17 KO cells. Cells were starved with EBSS for the indicated hours and immunoblotting was performed with the indicated antibodies.

Extended Data Figure 8

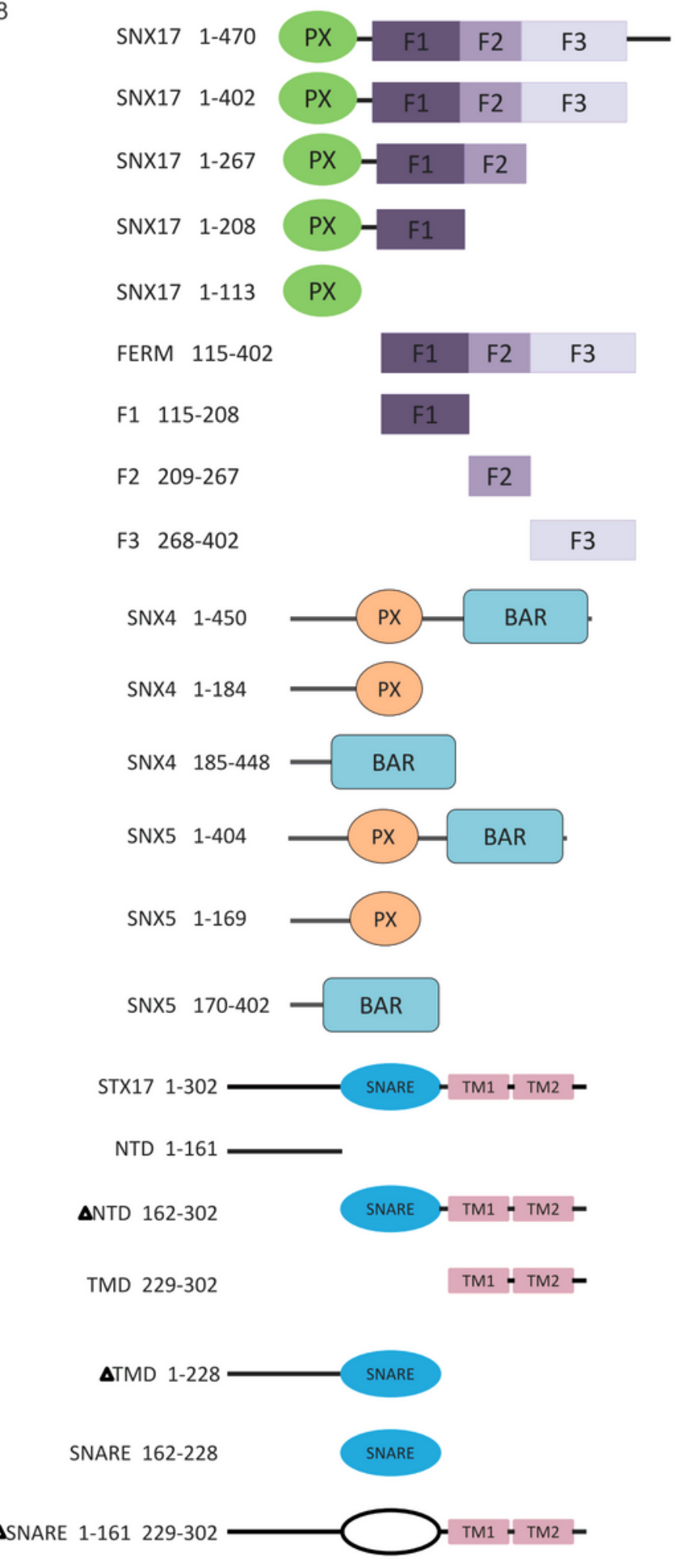

Page 38/39 
Figure 13

The truncated variants of STX17, SNX4, SNX5, and SNX17 used in this study 